\title{
POSITIVITY AND ALMOST POSITIVITY OF BIHARMONIC GREEN'S FUNCTIONS UNDER DIRICHLET BOUNDARY CONDITIONS
}

\author{
HANS-CHRISTOPH GRUNAU AND FRÉDÉRIC ROBERT
}

Dedicated to Prof. Wolf von Wahl on the occasion of his 65th birthday

\begin{abstract}
In general, for higher order elliptic equations and boundary value problems like the biharmonic equation and the linear clamped plate boundary value problem neither a maximum principle nor a comparison principle or - equivalently - a positivity preserving property is available. The problem is rather involved since the clamped boundary conditions prevent the boundary value problem from being reasonably written as a system of second order boundary value problems.

It is shown that, on the other hand, for bounded smooth domains $\Omega \subset$ $\mathbb{R}^{n}$, the negative part of the corresponding Green's function is "small" when compared with its singular positive part, provided $n \geq 3$.

Moreover, the biharmonic Green's function in balls $B \subset \mathbb{R}^{n}$ under Dirichlet (i.e. clamped) boundary conditions is known explicitly and is positive. It has been known for some time that positivity is preserved under small regular perturbations of the domain, if $n=2$. In the present paper, such a stability result is proved for $n \geq 3$.

Keywords: Biharmonic Green's functions, positivity, almost positivity, blowup procedure.
\end{abstract}

\section{INTRODUCTION}

Although simple examples show that strong maximum principles as satisfied e.g. by harmonic functions cannot hold true for solutions of higher order elliptic equations, it is reasonable to ask whether higher order boundary value problems may possibly enjoy a positivity preserving property. To be specific, we consider the clamped plate boundary value problem:

$$
\left\{\begin{array}{c}
\Delta^{2} u=f \text { in } \Omega, \\
u_{\left.\right|_{\partial \Omega}}=\frac{\partial}{\partial \nu} u_{\left.\right|_{\partial \Omega}}=0 .
\end{array}\right.
$$

Here $\Omega \subset \mathbb{R}^{n}$ is a bounded smooth domain with exterior unit normal $\nu$ at $\partial \Omega$, and $f$ is a sufficiently smooth datum. If $n=2$, the unknown $u$ models the vertical deflection of a horizontally clamped thin elastic plate from the horizontal equilibrium shape under the vertical load $f$. The boundary conditions $u_{\left.\right|_{\partial \Omega}}=\frac{\partial}{\partial \nu} u_{\left.\right|_{\partial \Omega}}=0$ are called Dirichlet boundary conditions and are natural in mechanics to model the horizontal clamping: More precisely, the condition $u_{\left.\right|_{\partial \Omega}}=0$ models the location of the clamping and the condition $\frac{\partial}{\partial \nu} u_{\partial \Omega}=0$ models the fact that the plate is clamped into some matter and is not able to rotate freely. We refer to the memoir written by Hadamard [17 for further considerations on this question. Throughout the present paper, always these boundary conditions will be considered. 
We shall discuss comparison principles or positivity preserving properties for the biharmonic operator. We say that the clamped plate boundary value problem enjoys a positivity preserving property in $\Omega$ if the following assertion holds:

for all $u \in C^{4}(\bar{\Omega})$ and $f \in C^{0}(\bar{\Omega})$ satisfying (1), one has that

$$
\{f \geq 0 \Rightarrow u \geq 0\} \text {. }
$$

This definition is the natural extension of the "positivity preserving property" for the harmonic operator, i.e. a comparison principle for a second-order operator. While the "positivity preserving property" is well understood for second-order operators, it is much more involved for fourth-order ones.

The positivity preserving property is closely related to the sign of the Green's function. More precisely, let $H_{\Omega}=H_{\Omega, \Delta^{2}}$ be the singular Green's function for the operator $\Delta^{2}$ in $\Omega$ under Dirichlet boundary conditions. Then, for any reasonable datum $f: \Omega \rightarrow \mathbb{R}$ the solution $u: \bar{\Omega} \rightarrow \mathbb{R}$ of the clamped plate boundary value problem (1) is given by

$$
u(x)=\int_{\Omega} H_{\Omega}(x, y) f(y) d y .
$$

In particular, the clamped plate boundary value problem enjoys a positivity preserving property in $\Omega$ iff $H_{\Omega}(x, y) \geq 0$ for all $x, y \in \Omega, x \neq y$.

It is important to remark that positivity issues are related to the specific kind of prescribed boundary conditions. More precisely, if one chooses Navier boundary condition (that is $u=\Delta u=0$ on $\partial \Omega$ ), then a twofold application of the second order comparison principle immediately yields a positivity preserving property. This simple situation is misleading in several respects: As we will explain below, counterexamples show that the situation is much more intricate for other boundary conditions. For Dirichlet boundary conditions which we consider here, this iterative trick fails completely. Moreover, even under Navier conditions, there is in general no positivity preserving property for perturbations of the biharmonic operator, see [29, 21], cf. also the general approach in [6].

The first example of a positive Green's function was given by Boggio [4 by means of a beautiful explicit formula for balls in $\mathbb{R}^{n}$, even for the Dirichlet problem for polyharmonic operators. Boggio [3] (1901) and Hadamard [17] (1908) conjectured that in arbitrary reasonable (two dimensional) domains $\Omega$, the positivity preserving property should hold true. In 1909 Hadamard [18 already knew, that the positivity conjecture is false in annuli with small inner radius. However, there was still some hope to prove positivity for convex domains.

Starting about 40 years later, numerous counterexamples disproved the BoggioHadamard conjecture, see e.g. 9, 11, 30. In particular, Coffman and Duffin 5] proved that in any two dimensional domain containing a right angle, the positivity preserving property does not hold. Even for smooth convex domains, the issue is quite intricate: Garabedian [11] (see also Shapiro-Tegmark [30] and HedenmalmJakobsson-Shimorin [19]) proved that for mildly eccentric ellipses, the prositivity preserving property does not hold true. On the other hand, according to [14, one has positivity in ellipses, which are close enough to a ball. For a more extensive survey and further references we also refer to $[14$. 
Therefore, the positivity preserving property does not hold true in general, even for arbitrarily smooth uniformly convex domains. Hence, it is important to understand the lack of "positivity preserving property" with the help of the Green's function. One may ask the following questions:

(1) Is positivity preserving in any bounded smooth domain possibly "almost true" in the sense that the negative part $H_{\Omega}^{-}(x, y):=\min \left\{H_{\Omega}(x, y), 0\right\}$ of the biharmonic Green's function under Dirichlet boundary conditions is "small relatively" to the singular positive part $H_{\Omega}^{+}(x, y)$ ?

(2) Are there are at least families of domains, different from balls, where the biharmonic Green's functions under Dirichlet boundary conditions are positive?

Question (1) is motivated by reactions of physicists and engineers on the mathematical results concerning positivity preserving and sign change. They may be summarised as follows: "For a clamped plate without corners, we do not expect downwards deflections if the force is pushing upwards. If such a phenomenon can be mathematically observed we think that perhaps the model is not perfectly suitable or that negativity is so small that it cannot be observed in reality." Also numerical experiments give support to the second hypothesis, which is subject of our first main result.

The general behaviour of the Green's functions is modeled on the behaviour of the singular fundamental solution on $\mathbb{R}^{n}$. On the whole space, we have that (letting $e_{n}$ be the $n$-dimensional volume of $B_{1}(0) \subset \mathbb{R}^{n}$ )

$$
H_{\mathbb{R}^{n}}(x, y)= \begin{cases}\frac{1}{2(n-4)(n-2) n e_{n}}|x-y|^{4-n}, & \text { when } n \geq 5, \\ \frac{1}{16 e_{4}} \log \frac{1}{|x-y|}, & \text { when } n=4, \\ -\frac{1}{8 \pi}|x-y|, & \text { when } n=3,\end{cases}
$$

for all $x, y \in \mathbb{R}^{n}, x \neq y$. If $n \geq 5$, this fundamental solution can even be interpreted as a Green's function in $\mathbb{R}^{n}$, where the Dirichlet boundary conditions at infinity are understood as a suitable decay. In the general framework of a bounded smooth domain of $\mathbb{R}^{n}$, Krasovskiı $[22$, 23. proved that there exists a constant $C(\Omega)$ such that

$$
\left|H_{\Omega}(x, y)\right| \leq C(\Omega) \begin{cases}|x-y|^{4-n}, & \text { if } n>4 \\ (1+|\log | x-y||), & \text { if } n=4 \\ 1, & \text { if } n<4\end{cases}
$$

for all $x, y \in \Omega, x \neq y$. These estimates give a uniform bound for the singular behaviour for Green's functions but do not consider their boundary behaviour. The latter was done by Dall'Acqua and Sweers $[8]$ by means of integrating Krasovskiî's 
estimates for $H$ and its derivatives.:

(2)

$$
\left|H_{\Omega}(x, y)\right| \leq \begin{cases}C \cdot|x-y|^{4-n} \min \left\{1, \frac{d(x)^{2} d(y)^{2}}{|x-y|^{4}}\right\}, & \text { if } n>4, \\ C \cdot \log \left(1+\frac{d(x)^{2} d(y)^{2}}{|x-y|^{4}}\right), & \text { if } n=4, \\ C \cdot d(x)^{2-n / 2} d(y)^{2-n / 2} \min \left\{1, \frac{d(x)^{n / 2} d(y)^{n / 2}}{|x-y|^{n}}\right\}, & \text { if } n<4 .\end{cases}
$$

Here, $d(x)=\mathrm{d}(x, \partial \Omega)$ and $C=C(\Omega)>0$ denotes a constant.

As far as the positive part $H_{\Omega}^{+}$is concerned, these estimates cannot be improved, see e.g. [15. However, they do not distinguish between the positive and the negative part of the Green's function. A distinction between $H_{\Omega}^{+}$and $H_{\Omega}^{-}$and showing that pairs of points of negativity cannot approach each other is the subject of our first main result, which was announced in [13].

Theorem 1. Let $\Omega \subset \mathbb{R}^{n}(n \geq 3)$ be a bounded $C^{4, \alpha}$-smooth domain. We denote by $H_{\Omega}$ the biharmonic Green's function in $\Omega$ under Dirichlet boundary conditions. Then, there exists a constant $\delta=\delta(\Omega)>0$ such that for any two points $x, y \in \Omega$, $x \neq y$,

$$
H_{\Omega}(x, y) \leq 0 \text { implies that }|x-y| \geq \delta .
$$

Consequently, there exists a constant $C=C(\Omega)>0$ such that for any two points $x, y \in \Omega, x \neq y$, we have that

$$
H_{\Omega}(x, y) \geq-C(\Omega)
$$

i.e. the negative part $H_{\Omega}^{-}$of the Green's function is bounded. Moreover, if $\Omega$ is smooth enough for (2) to hold true, the estimate (3) from below can be refined:

$$
H_{\Omega}(x, y) \geq-C(\Omega) d(x)^{2} d(y)^{2} .
$$

In other words: Around the pole, biharmonic Green's functions are always positive, if $n \geq 3$. And this behaviour is uniform, even if the pole approaches the boundary.

The proof of Theorem 1 indicates that one may not expect the full result to hold true also for $n=2$. The bound (4), however, was proved for the case $n=2$ and sufficiently smooth domains by Dall'Acqua, Meister and Sweers [7. Even for $n=2,3$, where the Green's function is bounded, (4) is a strong statement because in the case, where $x$ or $y$ is closer to the boundary than they are to each other, (2) would only give $H_{\Omega} \geq-C \frac{d(x)^{2} d(y)^{2}}{|x-y|^{n}}$. In this sense, (44) gains a factor of order $|x-y|^{n}$.

In his counterexample to positivity mentioned above, Garabedian [11 found in a mildly eccentric ellipse $\Omega$ opposite boundary points $x_{0}, y_{0} \in \partial \Omega$ with $\Delta_{x} \Delta_{y} H_{\Omega}\left(x_{0}, y_{0}\right)<$ 0 . This shows that qualitatively, the estimate (4) is sharp.

Another consequence of Theorem 1 is that one has a strong control of the negative part of solutions $u$ of the clamped plate boundary value problem (11) with nonnegative datum $f$ irrespective of the space dimension:

$$
f \geq 0 \Rightarrow\left\|u^{-}\right\|_{L^{\infty}(\Omega)} \leq C(\Omega)\|f\|_{L^{1}(\Omega)} .
$$

This estimate should be compared with the estimate for the full function: It follows from general elliptic theory [1] that for all $p>\frac{n}{4}$, there exists $C(p, \Omega)>0$ such 
that for solutions $u$ of the clamped plate boundary value problem (1) with datum $f$, we have that

$$
\|u\|_{L^{\infty}(\Omega)} \leq C(p, \Omega)\|f\|_{L^{p}(\Omega)} .
$$

Consequently, on the one hand, there might be a negative part for $u$, even if $f \geq 0$. But on the other hand, this negative part enjoys a nice strong control by a very weak norm. Therefore, although one has a lack of positivity in general, in this sense it is "small".

Let us now turn to Question (2): Are there at least families of domains, different from balls, where the biharmonic Green's functions under Dirichlet boundary conditions are positive? For two dimensional domains, this question was addressed in [14. There, it was shown that in domains $\Omega \subset \mathbb{R}^{2}$ being sufficiently close in $C^{4}$ sense to the (unit) disk $B \subset \mathbb{R}^{2}$, the biharmonic Green's function (under Dirichlet boundary conditions) is positive. Recently, Sassone [26] could relax the assumption on the domains to be close to $B$ in $C^{2, \alpha}$-sense. The authors could take advantage of conformal maps and the Riemann mapping theorem, pulling back the clamped plate boundary value problem to Dirichlet problems in the unit disk with the biharmonic operator as principal part and only with (small) lower order perturbations. The latter were treated in $B \subset \mathbb{R}^{n}$ ( $n$ arbitrary) in [15. The methods of [14, however, do not carry over to higher dimensions due to a lack of sufficiently many conformal maps. So, the question, whether the positivity of the biharmonic Green's function in the unit ball $B \subset \mathbb{R}^{n}$ is stable under domain perturbations, was left open.

This question is addressed in our next result. Assuming $n>2$, we show that in domains $\Omega \subset \mathbb{R}^{n}$, which are sufficiently close to the unit ball in a suitable $C^{4, \alpha}$-sense, the biharmonic Green's function under Dirichlet boundary conditions is indeed positive. More precisely, we prove the following theorem, where $I d$ denotes the identity map:

Theorem 2. Let $B$ be a unit ball of $\mathbb{R}^{n}, n \geq 3$. Then, there exists $\varepsilon_{0}=\varepsilon_{0}(n)>0$ such that the following holds true:

We assume that $\Omega \subset \mathbb{R}^{n}$ is a $C^{4, \alpha}$-smooth domain which is $\varepsilon_{0}$-close to the ball $B$ in the $C^{4, \alpha}$-sense, i.e.:

There exists a surjective $C^{4, \alpha}$-map $\psi: \bar{B} \rightarrow \bar{\Omega}$ such that

$\|I d-\psi\|_{C^{4, \alpha}(\bar{B})} \leq \varepsilon_{0}$.

Then, the Green's function $H_{\Omega}$ for $\Delta^{2}$ in $\Omega$ under Dirichlet boundary conditions is strictly positive:

$$
\forall x, y \in \Omega, \quad x \neq y: \quad H_{\Omega}(x, y)>0 .
$$

Assuming $\varepsilon_{0}$ small enough, this notion of closeness implies that there is a fixed neighbourhood $U$ of $\bar{B}, C^{4, \alpha}$-smooth injective extensions $\psi: U \rightarrow \mathbb{R}^{n}$, $\| I d-$ $\psi \|_{C^{4, \alpha}(U)}=O(\varepsilon)$ and $C^{4, \alpha}$-smooth inverse maps $\phi=\psi^{-1}: \psi(U) \rightarrow U$ such that $\psi(\bar{B})=\bar{\Omega}, \psi(B)=\Omega$.

For $n=2$, a direct and explicit proof based on perturbation series, Green's function estimates and conformal maps was given in [14, 15. This means that there, in principle, $\varepsilon_{0}$ may be calculated explicitly. Moreover, in the case $n=2$, closeness has to be assumed only in a weaker norm, see [26].

Here, the proof is somehow more indirect since a number of proofs by contradiction is involved so that it will be impossible to calculate $\varepsilon_{0}$ from our proofs. 
Furthermore, we have to make extensive use of general elliptic theory as provided by Agmon, Douglis and Nirenberg [1. We emphasize that Theorem 2 is by no means just a continuous dependence on data result, since the involved Green's "functions" are not simply functions but families of functions depending on the position of the singularity.

The problem consists in gaining uniformity with respect to the position of the singularity: When the singularity is in the interior, it is possible to use the local positivity results of Grunau-Sweers [16. But when the singularity approaches the boundary, the situation becomes more intricate and we develop a new technique for this problem. These remarks apply also to Theorem 1 which is proved by means of the same methods.

It remains as an interesting question to find out an optimal notion of closeness for a result like Theorem 2 to hold true. One might expect that like in the two-dimensional case (see 26] $C^{2, \alpha}$-closeness could suffice: Does the boundary curvature govern the positivity behaviour of biharmonic Green's functions? However, such a result would require new ideas and techniques; its possible proof would certainly be much more technical than ours. Sassone's approach is strictly twodimensional because of his use of conformal maps.

Our methods and techniques are general and our results can be extended to more general fourth order "positive definite self adjoint" elliptic operators under Dirichlet boundary conditions, where the principal part is a square of second order elliptic operators, and also to similar elliptic operators of higher order $2 m$ in dimensions $n \geq 2 m-1$ with reasonable boundary conditions of the type discussed in AgmonDouglis-Nirenberg [1].

\section{A MORE GENERAL RESUlt}

In order to prove Theorem 2, below in Theorem 3 we describe the possible situations how transition from positivity to sign change may occur within a smooth family of domains. It is then easy to see that none of these situations occurs in the (unit) ball in $\mathbb{R}^{n}, n>2$. Moreover, a special case of Theorem 3 will directly yield the proof of Theorem 1 .

To provide a more flexible result in Theorem 3, we will also include lower order perturbations. The formulation is somehow technical and requires in particular the notion of smooth domain perturbations, which we make precise in the following definition.

Definition 1. Let $\Omega,\left(\Omega_{k}\right)_{k \in \mathbb{N}}$ be domains of $\mathbb{R}^{n}$. We say that $\left(\Omega_{k}\right)_{k \in \mathbb{N}}$ is a $C^{4, \alpha_{-}}$ smooth perturbation of the bounded $C^{4, \alpha}$-smooth domain $\Omega$, and we write

$$
\lim _{k \rightarrow+\infty} \Omega_{k}=\Omega
$$

if the following facts are satisfied:

(i) There exist $N \in \mathbb{N}, p_{1}, \ldots, p_{N} \in \partial \Omega, \delta>0$ and open subsets $\omega \subset \subset \Omega$, $\omega \subset \subset \omega_{0} \subset \subset \Omega_{k}$ such that

$$
\Omega \subset \omega \cup \bigcup_{i=1}^{N} B_{\delta}\left(p_{i}\right) ; \quad \Omega_{k} \subset \omega \cup \bigcup_{i=1}^{N} B_{\delta}\left(p_{i}\right) ;
$$


(ii) for any $i \in\{1, \ldots, N\}$, there exists an open subset of $U_{i} \subset \mathbb{R}^{n}$ such that $0 \in U_{i}$, and a $C^{4, \alpha}$-smooth diffeomorphism $\Phi_{i}: U_{i} \rightarrow B_{2 \delta}\left(p_{i}\right)$ such that $\Phi_{i}(0)=p_{i}$ and

$\Phi_{i}\left(U_{i} \cap\left\{x_{1}<0\right\}\right)=\Phi_{i}\left(U_{i}\right) \cap \Omega, \Phi_{i}\left(U_{i} \cap\left\{x_{1}=0\right\}\right)=\Phi_{i}\left(U_{i}\right) \cap \partial \Omega ;$

(iii) for any $i \in\{1, \ldots, N\}$ and $k \in \mathbb{N}$, there exists $\Phi_{k, i}: U_{i} \rightarrow B_{2 \delta}\left(p_{i}\right)$ such that $\Phi_{k, i}\left(U_{i}\right)$ is an open subset, $B_{3 \delta / 2}\left(p_{i}\right) \subset \Phi_{k, i}\left(U_{i}\right)$ and $\Phi_{k, i}: U_{i} \rightarrow \Phi_{k, i}\left(U_{i}\right)$ is a $C^{4, \alpha}$-smooth diffeomorphism and

$\Phi_{k, i}\left(U_{i} \cap\left\{x_{1}<0\right\}\right)=\Phi_{k, i}\left(U_{i}\right) \cap \Omega_{k}, \Phi_{k, i}\left(U_{i} \cap\left\{x_{1}=0\right\}\right)=\Phi_{k, i}\left(U_{i}\right) \cap \partial \Omega_{k} ;$

(iv) for any $i \in\{1, \ldots, N\}, \lim _{k \rightarrow+\infty} \Phi_{k, i}=\Phi_{i}$ in $C_{l o c}^{4, \alpha}\left(U_{i}\right)$.

This definition implies that we have a well defined smooth exterior normal vector field so that $\Omega, \Omega_{k}, \partial \Omega$ and $\partial \Omega_{k}$ carry a canonical orientation. In what follows, the local charts will be chosen such that this orientation is observed, i.e. such that $\operatorname{Jac} \Phi_{i} \circ \Phi_{j}^{-1}>0$, Jac $\Phi_{k, i} \circ \Phi_{k, j}^{-1}>0$.

This definition covers in particular the following more special situation of smooth domain perturbation, which we make use of in proving Theorem 2, Let a sequence of mappings $\left(\psi_{k}\right)_{k \in \mathbb{N}}$ be such that there exists an open subset of $U \subset \mathbb{R}^{n}$ and $\psi_{k}: U \rightarrow \mathbb{R}^{n}$ for all $k \in \mathbb{N}$. We assume that $\lim _{k \rightarrow+\infty} \psi_{k}=I d$ in $C_{\text {loc }}^{4, \alpha}(U)$. Let $\Omega \subset \subset U$ be a $C^{4, \alpha}$-smooth bounded subset of $\mathbb{R}^{n}$ and let $\Omega_{k}:=\psi_{k}(\Omega)$ for all $k \in \mathbb{N}$. Then the sequence $\left(\Omega_{k}\right)_{k \in \mathbb{N}}$ is a smooth perturbation of $\Omega$.

Employing the notion of smooth domain perturbation we are now able to formulate our key result (where Theorems 1 and 2 are a consequence of as it is explained at the end of Section 6 ):

Theorem 3. Let $n \geq 3$, and $\left(\Omega_{k}\right)_{k \in \mathbb{N}}$ be a $C^{4, \alpha}$-smooth perturbation of the bounded $C^{4, \alpha}$-smooth domain $\Omega$ in the sense of Definition 1 . We consider a sequence $\left(a_{k}\right)_{k \in \mathbb{N}} \in C^{0, \alpha}\left(U_{0}\right)$, where $\Omega \subset \subset U_{0}$ and assume that there exists $a_{\infty} \in C^{0, \alpha}\left(U_{0}\right)$ such that

$$
\lim _{k \rightarrow+\infty} a_{k}=a_{\infty} \text { in } C_{l o c}^{0, \alpha}\left(U_{0}\right) .
$$

We assume further that there exists $\lambda>0$ such that

$$
\int_{\Omega_{k}}\left((\Delta \varphi)^{2}+a_{k} \varphi^{2}\right) d x \geq \lambda \int_{\Omega_{k}} \varphi^{2} d x
$$

for all $\varphi \in C_{c}^{\infty}\left(\Omega_{k}\right)$ and all $k \in \mathbb{N}$. Let $G_{k}$ be the Green's function of $\Delta^{2}+a_{k}$ on $\Omega_{k}$, and $G$ be the Green's function of $\Delta^{2}+a_{\infty}$ on $\Omega$, all with Dirichlet boundary conditions.

Finally, we suppose that there exist two sequences $\left(x_{k}\right)_{k \in \mathbb{N}},\left(y_{k}\right)_{k \in \mathbb{N}}$ such that $x_{k}, y_{k} \in \Omega_{k}$ and

$$
G_{k}\left(x_{k}, y_{k}\right)=0 \text { for all } k \in \mathbb{N} .
$$

Up to a subsequence, let $x_{\infty}:=\lim _{k \rightarrow+\infty} x_{k}$ and $y_{\infty}:=\lim _{k \rightarrow+\infty} y_{k}$. Then $x_{\infty}, y_{\infty} \in \bar{\Omega}, x_{\infty} \neq y_{\infty}$ and we are in one of the following situations:

(i) if $x_{\infty}, y_{\infty} \in \Omega$, then $G\left(x_{\infty}, y_{\infty}\right)=0$;

(ii) if $x_{\infty} \in \Omega$ and $y_{\infty} \in \partial \Omega$, then $\Delta_{y} G\left(x_{\infty}, y_{\infty}\right)=0$;

(iii) if $x_{\infty} \in \partial \Omega$ and $y_{\infty} \in \Omega$, then $\Delta_{x} G\left(x_{\infty}, y_{\infty}\right)=0$;

(iv) if $x_{\infty}, y_{\infty} \in \partial \Omega$, then $\Delta_{x} \Delta_{y} G\left(x_{\infty}, y_{\infty}\right)=0$. 
In the above statement, $\Delta_{x} G$ denotes the Laplacian with respect to the first variables, and $\Delta_{y} G$ denotes the Laplacian with respect to the second variables. The uniform coercivity assumption (5) is e.g. implied by a sign condition like $a_{k} \geq$ 0 and uniform smoothness of the domains to have a uniform Poincaré-Friedrichs inequality.

A result like Theorem 3 seems to be not known if $n=2$ and we doubt whether it is correct there. At least, our method cannot be extended to this case. The proof of Lemma 6.3 in dimensions $n=3,4$ uses a Nehari-type local positivity result [16, 24 ] which is not available for $n=2$.

More general lower order "self adjoint" perturbations of the biharmonic operator may be covered by precisely the same techniques. However, here we prefer to stick to a relatively simple situation in order to avoid too many technical details.

In the one dimensional context (clamped bars), related and quite concrete results were obtained by Schröder [27, 28, 29].

In order to gain a better feeling for the statement of Theorem 3 one should think of deforming the ball, where we know that positivity preserving holds true, smoothly into a domain where the biharmonic Green's function is sign changing (e.g. a long thin ellipsoid). There is a "last" domain where one still has a nonnegative Green's function. Our result describes the possible degeneracies of this positivity via which sign change occurs beyond this "last positivity-domain". The key statement is that $x_{\infty} \neq y_{\infty}$ so that it is impossible that negativity appears through the singularity: Around the singularity, our Green's functions are always positive. The most difficult part is to prove this also arbitrarily close to the boundary.

Alternatively one may think of the Green's function for $\Delta^{2}+\lambda$ in a ball for $\lambda \rightarrow \infty$ where again, initially one has positivity while sign changes occur for $\lambda \rightarrow \infty$ (see e.g. [2, 6]). We think that most likely the transition from positivity to sign change will occur via alternative (iv) of Theorem 3 .

Throughout the paper we assume that

$$
n \geq 3 \text {. }
$$

A first essential step in proving Theorem 3 consists in providing uniform bounds (in $k$ ) for the Green's functions like

$$
\left|G_{k}(x, y)\right| \leq C \cdot \begin{cases}|x-y|^{4-n}, & \text { if } n>4 \\ (1+|\log | x-y||), & \text { if } n=4 \\ 1, & \text { if } n=3\end{cases}
$$

Moreover, if $n=3$, 4 , the somehow irregular estimates for $G_{k}$ require to focus first on the gradients, where estimates like

$$
\left|\nabla G_{k}(x, y)\right| \leq C \cdot \begin{cases}|x-y|^{-1}, & \text { if } n=4, \\ 1, & \text { if } n=3,\end{cases}
$$

are available, which are compatible with the scaling arguments performed below. In this respect, the proof is more difficult in dimensions $n=3$ and in particular $n=4$. Estimates (6) and (7) are due to Krasovskiu 23], provided the family $\left(\Omega_{k}\right)$ is assumed to be uniformly $C^{11}$-smooth. This assumption is due to the great generality of the boundary value problems considered by Krasovskil. We prove in Theorem 4 that in our special situation, (6) and (7) hold true under our uniform $C^{4, \alpha}$-smoothness assumptions. Preliminary properties of the Green's functions are shown in Section 3. while Section 5 is devoted to convergence properties of families 
of Green's functions in $\left(\Omega_{k}\right)_{k \in \mathbb{N}}$. The proofs of Theorems 3 and, as a consequence, of Theorems 1 and 2 are finally given in Section 6 .

Notation. Straightening the boundary requires to work in $\mathbb{R}_{-}^{n}:=\left\{x \in \mathbb{R}^{n}\right.$ : $\left.x_{1}<0\right\}$, where we write $\mathbb{R}^{n} \ni x=\left(x_{1}, \bar{x}\right) . e_{n}$ denotes the $n$-dimensional volume of $B_{1}(0) \subset \mathbb{R}^{n} . C_{c}^{\infty}(\Omega)$ denotes the space of arbitrarily smooth functions with compact support in $\Omega$ and $\mathcal{D}^{\prime}(\Omega)$ its dual, i.e. the space of distributions on $\Omega$.

\section{The Green's Function $G$ For the Perturbed Biharmonic operator}

In the first part of this section, we consider a fixed operator $\Delta^{2}+a$ in a fixed smooth domain and construct and investigate the corresponding Green's function.

Proposition 3.1. Let $\Omega \subset \mathbb{R}^{n}$ be a bounded $C^{4, \alpha}$-smooth domain and $a \in C^{0, \alpha}(\bar{\Omega})$. We assume that $\Delta^{2}+a$ is coercive. Then, for every $x \in \Omega$, there exists a unique Green's function $G_{x} \in L^{1}(\Omega) \cap C^{4, \alpha}(\bar{\Omega} \backslash\{x\})$ such that $\left.G_{x}\right|_{\partial \Omega}=\left.\frac{\partial G_{x}}{\partial \nu}\right|_{\partial \Omega}=0$ and that for all $\varphi \in C^{4}(\bar{\Omega})$ with $\left.\varphi\right|_{\partial \Omega}=\left.\frac{\partial \varphi}{\partial \nu}\right|_{\partial \Omega}=0$ one has the following representation formula:

$$
\varphi(x)=\int_{\Omega} G_{x}(y)\left(\Delta^{2} \varphi(y)+a(y) \varphi(y)\right) d y .
$$

If $R>0$ is such that $\Omega \subset B_{R}(0)$ and $\lambda>0$ such that

$$
\forall \varphi \in W_{0}^{2,2}(\Omega): \int_{\Omega}\left((\Delta \varphi)^{2}+a \varphi^{2}\right) d y \geq \lambda \int_{\Omega} \varphi^{2} d y
$$

then, the following estimate for the Green's function holds true:

$$
\begin{aligned}
\left(\not\left|r_{x}(y)\right| \leq\right. & C\left(\lambda, R, n,\|a\|_{C^{0, \alpha}}, \Omega\right) \\
& \cdot \begin{cases}\left(|x-y|^{4-n}+\max \{d(x, \partial \Omega), d(y, \partial \Omega)\}^{4-n}\right), & \text { if } n>4, \\
1+|\log | x-y||+|\log (\max \{d(x, \partial \Omega), d(y, \partial \Omega)\})|, & \text { if } n=4, \\
1, & \text { if } n=3 .\end{cases}
\end{aligned}
$$

If $n=3,4$, we further prove the following gradient estimates:

$$
\begin{aligned}
(1 \nabla)_{(x, y)} G_{x}(y) \mid \leq & C\left(\lambda, R, n,\|a\|_{C^{0, \alpha}}, \Omega\right) \\
& \cdot \begin{cases}\left(|x-y|^{-1}+\max \{d(x, \partial \Omega), d(y, \partial \Omega)\}^{-1}\right), & \text { if } n=4, \\
1, & \text { if } n=3 .\end{cases}
\end{aligned}
$$

The dependence of the constants $C$ on $\Omega$ is explicit via the $C^{4, \alpha}$-properties of $\partial \Omega$.

Proof. We first prove extensively the generic case $n>4$. At the end we comment on the changes and additional arguments which have to be made, if $n=4$ or $n=3$.

Case $n>4$. We introduce the fundamental solution $\Gamma_{0}$ of the biharmonic operator

$$
\Gamma_{0}(x, y):=\frac{1}{2(n-4)(n-2) n e_{n}}|x-y|^{4-n}
$$

so that $\Gamma_{0} \in C^{\infty}(\bar{\Omega} \times \bar{\Omega}) \backslash\{(x, y): x=y\}$. We define recursively for $j \geq 0$

$$
\Gamma_{j+1}(x, y):=-\int_{\Omega} \Gamma_{j}(x, z) a(z) \Gamma_{0}(z, y) d z
$$


and have that $\Gamma_{j} \in C^{4, \alpha}(\bar{\Omega} \times \bar{\Omega} \backslash\{(x, y): x=y\})$ is well defined and, according to a Lemma of Giraud [12, that for $j \geq 1$

$$
\left|\Gamma_{j}(x, y)\right| \leq \begin{cases}C_{j}|x-y|^{4(j+1)-n}, & \text { if }(j+1)<\frac{n}{4}, \\ C_{j}(1+|\log | x-y||), & \text { if }(j+1)=\frac{n}{4}, \\ C_{j}, & \text { if }(j+1)>\frac{n}{4}\end{cases}
$$

Here, $C_{j}=C_{j}\left(n, R,\|a\|_{\infty}\right)$, where $R>0$ is chosen such that $\Omega \subset B_{R}(0)$. We fix some $\ell>\frac{n}{4}, x \in \Omega$ and for $u_{x} \in C^{4, \alpha}(\bar{\Omega})$ to be suitably determined below, we put

$$
G_{x}(y):=\Gamma_{0}(x, y)+\sum_{j=1}^{\ell} \Gamma_{j}(x, y)+u_{x}(y) .
$$

One should observe that $\sum_{j=0}^{\infty} \Gamma_{j}$ is the Neumann-series for the fundamental solution for the perturbed differential operator. We have that $G_{x} \in C^{4, \alpha}(\bar{\Omega} \backslash\{x\})$. In order that $G_{x}$ becomes indeed a Green's function for the Dirichlet problem for $\Delta^{2}+a$, i.e. that indeed formula (8) is satisfied, we need $u_{x}$ to be a solution of the following Dirichlet problem

$$
\begin{cases}\Delta^{2} u_{x}(y)+a(y) u_{x}(y)=-a(y) \Gamma_{\ell}(x, y) & \text { in } \Omega \\ u_{x}(y)=-\Gamma_{0}(x, y)-\sum_{j=1}^{\ell} \Gamma_{j}(x, y) & \text { for } y \in \partial \Omega \\ \frac{\partial}{\partial \nu} u_{x}(y)=-\frac{\partial}{\partial \nu_{y}} \Gamma_{0}(x, y)-\sum_{j=1}^{\ell} \frac{\partial}{\partial \nu_{y}} \Gamma_{j}(x, y) & \text { for } y \in \partial \Omega\end{cases}
$$

Since $\ell>\frac{n}{4}$, the right hand side $-a \cdot \Gamma_{\ell}(x,$.$) is Hölder continuous with Hölder norm$ bounded by a constant $C\left(n, R,\|a\|_{C^{0, \alpha}}\right)$. The $C^{1, \alpha}$-norm of the datum for $\left.u_{x}\right|_{\partial \Omega}$ and the $C^{0, \alpha}$-norm of the datum for $\left.\frac{\partial}{\partial \nu} u_{x}\right|_{\partial \Omega}$ are bounded by $C(n, R, \partial \Omega) d(x, \partial \Omega)^{3-n-\alpha}$. The dependence of the constant $C$ on $\partial \Omega$ is in principle constructive and explicit via its curvature properties and their derivatives. According to $C^{1, \alpha}$-estimates for boundary value problems in variational form like (14) - see [1, Thm. 9.3] - we see that

$$
\left\|u_{x}\right\|_{C^{1, \alpha}(\bar{\Omega})} \leq C\left(n, R, \lambda,\|a\|_{C^{0, \alpha}}, \partial \Omega\right) d(x, \partial \Omega)^{3-n-\alpha} .
$$

One should observe that the differential operators are uniformly coercive, so that no $u_{x}$-terms need to appear on the right-hand-side.

As long as $d(y, \partial \Omega) \leq d(x, \partial \Omega)$, (15) shows that

$$
\left|u_{x}(y)\right| \leq C\left(C_{0}, n, R, \lambda,\|a\|_{C^{0, \alpha}}, \partial \Omega\right) d(x, \partial \Omega)^{4-n}
$$

and hence

$$
\left|G_{x}(y)\right| \leq C\left(C_{0}, n, R, \lambda,\|a\|_{C^{0, \alpha}}, \partial \Omega\right)\left(|x-y|^{4-n}+d(x, \partial \Omega)^{4-n}\right) .
$$

If $d(y, \partial \Omega)>d(x, \partial \Omega)$ we conclude from (16) by exploiting the symmetry of the Green's function:

$$
\left|G_{x}(y)\right|=\left|G_{y}(x)\right| \leq C\left(C_{0}, n, R, \lambda,\|a\|_{C^{0, \alpha}}, \partial \Omega\right)\left(|x-y|^{4-n}+d(y, \partial \Omega)^{4-n}\right) .
$$

Combining (16) and (17) yields (9) for $n>4$.

Case $n=4$. Here the fundamental solution we start with is

$$
\Gamma_{0}(x, y):=-\frac{1}{16 e_{4}} \log |x-y| \text {. }
$$


We proceed with the iterated kernels $\Gamma_{j}$. In view of the mild singularity of $\Gamma_{0}$, however, it is sufficient to choose $\ell=1$. As above we find that

$$
\left\|u_{x}\right\|_{C^{1, \alpha}(\bar{\Omega})} \leq C\left(n, R, \lambda,\|a\|_{C^{0, \alpha}}, \partial \Omega\right) d(x, \partial \Omega)^{-1-\alpha} .
$$

As long as $d(y, \partial \Omega) \leq d(x, \partial \Omega)$, (19) shows that

$$
\left|\nabla_{y} G_{x}(y)\right| \leq C\left(C_{0}, n, R, \lambda,\|a\|_{C^{0, \alpha}}, \partial \Omega\right)\left(|x-y|^{-1}+d(x, \partial \Omega)^{-1}\right) .
$$

In order to exploit the symmetry of $G_{x}(y)$ we need a similar estimate also for $\left|\nabla_{x} G_{x}(y)\right|$. To this end one has to differentiate (14) with respect to the parameter (!) $x$ and obtains as before that for $d(y, \partial \Omega) \leq d(x, \partial \Omega)$

$$
\left|\nabla_{x} G_{x}(y)\right| \leq C\left(C_{0}, n, R, \lambda,\|a\|_{C^{0, \alpha}}, \partial \Omega\right)\left(|x-y|^{-1}+d(x, \partial \Omega)^{-1}\right) .
$$

By symmetry $G_{x}(y)=G_{y}(x)$, and (21) shows that for $d(x, \partial \Omega) \leq d(y, \partial \Omega)$, one has

$$
\left|\nabla_{y} G_{x}(y)\right| \leq C\left(C_{0}, n, R, \lambda,\|a\|_{C^{0, \alpha}}, \partial \Omega\right)\left(|x-y|^{-1}+d(y, \partial \Omega)^{-1}\right)
$$

while (20) yields

$$
\left|\nabla_{x} G_{x}(y)\right| \leq C\left(C_{0}, n, R, \lambda,\|a\|_{C^{0, \alpha}}, \partial \Omega\right)\left(|x-y|^{-1}+d(y, \partial \Omega)^{-1}\right) .
$$

Combining (20)-(23) proves (10) and hence (9) in the case $n=4$.

Case $n=3$. Here, we simply work with the bounded Lipschitz continuous fundamental solution

$$
\Gamma_{0}(x, y):=-\frac{1}{8 \pi}|x-y|
$$

so that no iterative procedure is needed and we may directly work with $\ell=0$. One comes up with

$$
\left\|u_{x}\right\|_{C^{1, \alpha}(\bar{\Omega})} \leq C\left(R, n, \lambda,\|a\|_{C^{0, \alpha}}, \partial \Omega\right) d(x, \partial \Omega)^{-\alpha} .
$$

Proceeding as for $n=4$ yields (10) and hence (9) also in the case $n=3$.

Let us now show that assuming certain uniform estimates on the Green's functions $H_{k}$ for the biharmonic operators on a family $\left(\Omega_{k}\right)$ of domains according to Definition 1 implies similar uniform estimates for the Green's functions of the perturbed biharmonic operators $\Delta^{2}+a_{k}$ on $\Omega_{k}$ :

Proposition 3.2. Let $n \geq 4$ and $\left(\Omega_{k}\right)_{k \in \mathbb{N}}$ be a $C^{4, \alpha}$-smooth perturbation of the bounded $C^{4, \alpha}$-smooth domain $\Omega$ according to Definition 1 and $R>0$ such that $\Omega_{k} \subset B_{R}(0)$. Let $H_{k} \in C^{4}\left(\overline{\Omega_{k}} \times \overline{\Omega_{k}} \backslash\{(x, y): x=y\}\right)$ denote the Green's functions for $\Delta^{2}$ in $\Omega_{k}$ and assume that there exists a uniform constant $C_{1}$ such that for all $k$ and all $x, y \in \Omega_{k}(x \neq y)$

$$
\left|H_{k}(x, y)\right| \leq C_{1} \cdot \begin{cases}|x-y|^{4-n}, & \text { if } n>4, \\ (1+|\log | x-y||), & \text { if } n=4 .\end{cases}
$$

Let $a_{k} \in C^{0, \alpha}\left(\overline{\Omega_{k}}\right)$ and $\Lambda>0$ such that $\forall k:\left\|a_{k}\right\|_{C^{0, \alpha}\left(\overline{\Omega_{k}}\right)} \leq \Lambda$ and let $\lambda>0$ be such that

$$
\forall k \quad \forall \varphi \in C_{c}^{\infty}\left(\Omega_{k}\right): \quad \int_{\Omega_{k}}\left((\Delta \varphi)^{2}+a_{k} \varphi^{2}\right) d y \geq \lambda \int_{\Omega_{k}} \varphi^{2} d y .
$$


We denote by $G_{k}$ the Green's functions for $\Delta^{2}+a_{k}$ in $\Omega_{k}$. Then, there exists a constant $C_{2}=C_{2}\left(R, n, C_{1}, \lambda, \Lambda, \Omega\right)$ such that one has the following estimate:

$$
\forall x, y \in \Omega_{k}, x \neq y: \quad\left|G_{k}(x, y)\right| \leq C_{2} \cdot \begin{cases}|x-y|^{4-n}, & \text { if } n>4, \\ (1+|\log | x-y||), & \text { if } n=4 .\end{cases}
$$

Moreover, assuming

$$
\forall x, y \in \Omega_{k}, x \neq y: \quad\left|\nabla_{(x, y)} H_{k}(x, y)\right| \leq C_{1}|x-y|^{-1}, \quad \text { if } n=4,
$$

in dimension $n=4$ implies that

$$
\forall x, y \in \Omega_{k}, x \neq y: \quad\left|\nabla_{(x, y)} G_{k}(x, y)\right| \leq C_{2}|x-y|^{-1}, \quad \text { if } n=4 .
$$

The dependence on $\left(\Omega_{k}\right)_{k}$ as regular perturbations of $\Omega$ is explicit via the geometric properties of $\partial \Omega$. As long as these properties are uniformly satisfied, the same constant may be chosen.

The case $n=3$ need not be covered here, since in this case, Proposition 3.1 already provides strong enough information for our purposes.

Proof. We proceed quite similarly as in the proof of Proposition 3.1, but now using the biharmonic Green's functions $H_{k}$ instead of $\Gamma_{0}$. That means that in $\Omega_{k}$, we define inductively

$$
\begin{gathered}
\Gamma_{k, 1}(x, y):=-\int_{\Omega_{k}} H_{k}(x, z) a_{k}(z) H_{k}(z, y) d z \\
\Gamma_{k, j+1}(x, y):=-\int_{\Omega_{k}} \Gamma_{k, j}(x, z) a_{k}(z) H_{k}(z, y) d z .
\end{gathered}
$$

Moreover, as above, we make the ansatz with $u_{k, x} \in C^{4, \alpha}\left(\overline{\Omega_{k}}\right)$

$$
G_{k}(x, y):=H_{k}(x, y)+\sum_{j=1}^{\ell} \Gamma_{k, j}(x, y)+u_{k, x}(y) .
$$

We choose $\ell>\frac{n}{4}+1$ so that

$$
\left|\Gamma_{k, \ell}\right| \leq C(R, n, \Lambda), \quad\left|\nabla \Gamma_{k, \ell}\right| \leq C(R, n, \Lambda),
$$

while for the other $\Gamma_{j}$, we have in particular that

$$
\left|\Gamma_{k, j}(x, y)\right| \leq C(R, n, \Lambda) \cdot \begin{cases}|x-y|^{4-n}, & \text { if } n>4, \\ (1+|\log | x-y||), & \text { if } n=4,\end{cases}
$$

and assuming (28) that

$$
\forall x, y \in \Omega_{k}, x \neq y: \quad\left|\nabla_{(x, y)} \Gamma_{k, j}(x, y)\right| \leq C(R, n, \Lambda) \cdot|x-y|^{-1}, \text { if } n=4 .
$$

As before we see that $G_{k}$ is indeed the Green's function for the Dirichlet problem for $\Delta^{2}+a_{k}$ in $\Omega_{k}$, iff the $u_{k, x}$ solve the following boundary value problems:

$$
\begin{cases}\Delta^{2} u_{k, x}(y)+a_{k}(y) u_{k, x}(y)=-a_{k}(y) \Gamma_{k, \ell}(x, y) & \text { in } \Omega_{k} \\ u_{k, x}(y)=\frac{\partial}{\partial \nu} u_{k, x}=0 & \text { for } y \in \partial \Omega_{k} .\end{cases}
$$

The right hand side is uniformly bounded, the operators are uniformly coercive. Hence, $L^{p}$-theory (see [1]) combined with Sobolev embedding theorems and differentiating (34) with respect to the parameter $x$ yields

$$
\begin{aligned}
\left|u_{k, x}(y)\right| & \leq C\left(R, n, C_{1}, \lambda, \Lambda,\left(\Omega_{k}\right)_{k \in \mathbb{N}}\right), \\
\left|\nabla_{(x, y)} u_{k, x}(y)\right| & \leq C\left(R, n, C_{1}, \lambda, \Lambda,\left(\Omega_{k}\right)_{k \in \mathbb{N}}\right) .
\end{aligned}
$$


The dependence on $\left(\Omega_{k}\right)_{k}$ is uniform in the sense described before the present proof. Inserting (31), (32), (35) and (33) into (30) proves the claim.

Finally, we need a more precise statement concerning the smoothness of the Green's functions simultaneously with respect to both variables.

Proposition 3.3. Under the assumptions of Proposition 3.2 we have in addition that

$$
G_{k} \in C^{4, \alpha}\left(\overline{\Omega_{k}} \times \overline{\Omega_{k}} \backslash\{(x, y): x \neq y\}\right) .
$$

Proof. We let $i \in\{0, \ldots, 3\}$ and $p \in(n, n+1)$ so that in particular $4-i-\frac{n}{p}>0$. We let $\varphi \in C_{c}^{\infty}\left(\Omega_{k}\right)$ and consider $\psi \in C^{4, \alpha}\left(\overline{\Omega_{k}}\right)$ such that $\Delta^{2} \psi+a_{k} \psi=\varphi$ in $\Omega_{k}$ and $\psi=\partial_{\nu} \psi=0$ on $\partial \Omega_{k}$. It follows from regularity theory (see [1]) and Sobolev's embedding theorem that

$$
\|\psi\|_{C^{i, \beta}\left(\overline{\Omega_{k}}\right)} \leq C\|\psi\|_{W^{4, p}\left(\Omega_{k}\right)} \leq C\|\varphi\|_{L^{p}\left(\Omega_{k}\right)}
$$

with $\beta \leq 4-i-\frac{n}{p}, \beta \in(0,1)$. Here $W^{4, p}$ denotes the Sobolev space of order 4 in differentiability and of order $p$ in integrability. Since $\psi(x)=\int_{\Omega_{k}} G_{k}(x, y) \varphi(y) d y$, we get that $\nabla_{x}^{i} G_{k}$ makes sense and that

$$
\left|\int_{\Omega_{k}}\left(\nabla_{x}^{i} G_{k}(x, y)-\nabla_{x}^{i} G_{k}\left(x^{\prime}, y\right)\right) \varphi(y) d y\right| \leq C_{2}\|\varphi\|_{L^{p}\left(\Omega_{k}\right)}\left|x-x^{\prime}\right|^{\beta} .
$$

By duality, we then get that $y \mapsto \nabla_{x}^{i} G_{k}(x, y) \in L^{q}\left(\Omega_{k}\right)$ for all $q \in\left(\frac{n+1}{n}, \frac{n}{n-1}\right)$ and that

$$
\left\|\nabla_{x}^{i} G_{k}(x, \cdot)-\nabla_{x}^{i} G_{k}\left(x^{\prime}, \cdot\right)\right\|_{q} \leq C(q)\left|x-x^{\prime}\right|^{\beta} \text { for all } x, x^{\prime} \in \Omega_{k} .
$$

It follows from the equation satisfied by $G_{k}(x, \cdot)$ that we have $\Delta^{2} \nabla_{x}^{i} G_{k}(x, \cdot)+$ $a \nabla_{x}^{i} G_{k}(x, \cdot)=0$ in $\mathcal{D}^{\prime}\left(\Omega_{k} \backslash\{x\}\right)$ and $\nabla_{x}^{i} G_{k}(x, \cdot)=0, \partial_{\nu} \nabla_{x}^{i} G_{k}(x, \cdot)=0$ on $\partial \Omega_{k}$. It then follows from regularity theory that $\nabla_{x}^{i} G_{k}(x, \cdot) \in C^{4, \alpha}\left(\overline{\Omega_{k}} \backslash\{x\}\right)$. Moreover, for all $\delta>0$, there exists $C(\delta)>0$ such that

$$
\left\|\nabla_{x}^{i} G_{k}(x, \cdot)-\nabla_{x}^{i} G_{k}\left(x^{\prime}, \cdot\right)\right\|_{C^{4, \alpha}\left(\overline{\Omega_{k}} \backslash\left(B_{\delta}(x) \cup B_{\delta}\left(x^{\prime}\right)\right)\right)} \leq C(\delta)\left|x-x^{\prime}\right|^{\beta}
$$

for all $x, x^{\prime} \in \Omega_{k}$. This is valid for $i \leq 3$; using the symmetry of the Green's function, we have a similar result for $i=4$ with respect to the $C^{3, \alpha}\left(\overline{\Omega_{k}} \backslash\left(B_{\delta}(x) \cup B_{\delta}\left(x^{\prime}\right)\right)\right)$ norm. It then follows that all derivatives of order 4 are covered so that $G_{k} \in$ $C^{4, \alpha}\left(\overline{\Omega_{k}} \times \overline{\Omega_{k}} \backslash\left\{(x, x): x \in \overline{\Omega_{k}}\right\}\right)$. This proves the proposition.

\section{Uniform BOUNDS FOR the GREEN'S FUnCTIONS}

As before, we consider a family of bounded regular domains $\left(\Omega_{k}\right)_{k \in \mathbb{N}}$ being a smooth perturbation of a fixed bounded regular domain $\Omega$ according to Definition 1 . We focus on proving

$$
\begin{gathered}
\left|H_{k}(x, y)\right| \leq C_{1}\left\{\begin{array}{lr}
|x-y|^{4-n}, & \text { if } n>4, \\
(1+|\log | x-y||), & \text { if } n=4, \\
1, & \text { if } n=3 ;
\end{array}\right. \\
\left|\nabla_{(x, y)} H_{k}(x, y)\right| \leq C_{1} \begin{cases}|x-y|^{-1}, & \text { if } n=4, \\
1, & \text { if } n=3 ;\end{cases}
\end{gathered}
$$

with the constant $C_{1}=C_{1}(\Omega)$ being uniform for the whole family $\left(\Omega_{k}\right)_{k \in \mathbb{N}}$. Originally, this type of estimates on the Green's functions is due to Krasovskiu [23] (cf. 
also 22. ) even for very general boundary value problems for even order elliptic operators. For the reader's convenience, we include here an independant and shorter proof of these estimates.

Theorem 4. Let $\Omega$ be a bounded $C^{4, \alpha}$-smooth domain of $\mathbb{R}^{n}, n \geq 3$ and $\left(\Omega_{k}\right)_{k \in \mathbb{N}}$ a $C^{4, \alpha}$-smooth perturbation of $\Omega$. We denote by $H_{k}$ the Green's functions for $\Delta^{2}$ in $\Omega_{k}$ under Dirichlet boundary conditions.

Then, there exists a constant $C_{1}>0$ such that for all $k$ and all $x, y \in \Omega_{k}$ with $x \neq y$ one has that

$$
\left|H_{k}(x, y)\right| \leq C_{1} \cdot \begin{cases}|x-y|^{4-n}, & \text { if } n>4 \\ (1+|\log | x-y||), & \text { if } n=4 \\ 1, & \text { if } n=3 .\end{cases}
$$

Moreover, for $n=3,4$ we prove that

$$
\forall x, y \in \Omega_{k}, x \neq y: \quad\left|\nabla_{(x, y)} H_{k}(x, y)\right| \leq C_{1} \cdot \begin{cases}|x-y|^{-1}, & \text { if } n=4, \\ 1, & \text { if } n=3 .\end{cases}
$$

Proof. If $n=3$, the statement of Proposition 3.1 is already strong enough and nothing remains to be proved. We postpone the case $n=4$ and start with proving the theorem in the generic case $n>4$. We argue by contradiction and assume that there exist two sequences $\left(x_{k}\right)_{k \in \mathbb{N}},\left(y_{k}\right)_{k \in \mathbb{N}}$ with $x_{k}, y_{k} \in \Omega_{\ell_{k}}$ such that $x_{k} \neq y_{k}$ for all $k \in \mathbb{N}$ and such that

$$
\lim _{k \rightarrow+\infty}\left|x_{k}-y_{k}\right|^{n-4}\left|H_{\ell_{k}}\left(x_{k}, y_{k}\right)\right|=+\infty .
$$

It is enough to consider $\ell_{k}=k$; other situations may be reduced to this by relabelling or are even more special. After possibly passing to a subsequence, it follows from (9) that there exists $x_{\infty} \in \partial \Omega$ such that

$$
\lim _{k \rightarrow+\infty} x_{k}=x_{\infty} \text { and } \lim _{k \rightarrow+\infty} \frac{d\left(x_{k}, \partial \Omega_{k}\right)}{\left|x_{k}-y_{k}\right|}=0 .
$$

We remark that the constant in (9) can be chosen uniformly for the family $\left(\Omega_{k}\right)_{k \in \mathbb{N}}$.

Lemma 4.1. Assume that $n \geq 4$. For any $q \in\left(\frac{n}{n-3}, \frac{n}{n-4}\right)$, there exists $C(q)>0$ such that for all $k$ and all $x \in \Omega_{k}$ we have

$$
\left\|H_{k}(x, .)\right\|_{L^{q}\left(\Omega_{k}\right)} \leq C d\left(x, \partial \Omega_{k}\right)^{4-n+\frac{n}{q}} .
$$

The constant $C$ can be chosen uniformly for the family $\left(\Omega_{k}\right)_{k \in \mathbb{N}}$.

Proof. We proceed with the help of a duality argument. Let $\psi \in C_{c}^{\infty}\left(\Omega_{k}\right)$ and let $\varphi \in C^{4, \alpha}\left(\overline{\Omega_{k}}\right)$ be a solution of

$$
\begin{cases}\Delta^{2} \varphi=\psi & \text { in } \Omega_{k} \\ \varphi=\partial_{\nu} \varphi=0 & \text { on } \partial \Omega_{k}\end{cases}
$$

Let $q \in\left(\frac{n}{n-3}, \frac{n}{n-4}\right)$ and denote $q^{\prime}=\frac{q}{q-1}$ the dual exponent, so that in particular $\frac{n}{4}<q^{\prime}<\frac{n}{3}$. It follows from elliptic estimates [1, Thm. 15.2] that there exists $C_{3}>0$ independent of $\varphi, \psi$ and $k$ such that

$$
\|\varphi\|_{W^{4, q^{\prime}}\left(\Omega_{k}\right)} \leq C_{3}\|\psi\|_{L^{q^{\prime}}\left(\Omega_{k}\right)} .
$$


The embedding $W^{4, q^{\prime}}\left(\Omega_{k}\right) \hookrightarrow C^{0, \beta}\left(\overline{\Omega_{k}}\right)$ with $\beta=4-\frac{n}{q^{\prime}}=4-n+\frac{n}{q}$ being continuous uniformly in $k$ shows that there exists $C_{4}>0$ independent of $\varphi$ and $k$ such that $\|\varphi\|_{C^{0, \beta}\left(\overline{\Omega_{k}}\right)} \leq C_{4}\|\varphi\|_{W^{4, q^{\prime}}\left(\Omega_{k}\right)}$. Let $x \in \Omega_{k}$ and $x^{\prime} \in \partial \Omega_{k}$. We then get that

$$
|\varphi(x)|=\left|\varphi(x)-\varphi\left(x^{\prime}\right)\right| \leq\|\varphi\|_{C^{0, \beta}\left(\overline{\Omega_{k}}\right)}\left|x-x^{\prime}\right|^{\beta} \leq C_{3} C_{4}\|\psi\|_{L^{q^{\prime}}\left(\Omega_{k}\right)}\left|x-x^{\prime}\right|^{\beta} .
$$

Moreover, $\varphi(x)=\int_{\Omega_{k}} H_{k}(x, y) \psi(y) d y$ for all $x \in \Omega_{k}$ by Green's representation formula. Therefore, taking the infimum with respect to $x^{\prime} \in \partial \Omega_{k}$, we have that

$$
\left|\int_{\Omega_{k}} H_{k}(x, y) \psi(y) d y\right| \leq C_{3} C_{4}\|\psi\|_{L^{q^{\prime}}\left(\Omega_{k}\right)} d\left(x, \partial \Omega_{k}\right)^{\beta}
$$

for all $\psi \in C_{c}^{\infty}\left(\Omega_{k}\right)$. Inequality (40) then follows.

Lemma 4.2. Assuming $n>4$ and (38), one has that $\lim _{k \rightarrow+\infty}\left|x_{k}-y_{k}\right|=0$.

Proof. Assume by contradiction that $\left|x_{k}-y_{k}\right|$ does not converge to 0 . After extracting a subsequence we may then assume that there exists $\delta>0$ such that all $x_{k} \in B_{\delta}\left(x_{\infty}\right)$ and all $y_{k} \in \Omega_{k} \backslash \overline{B_{3 \delta}\left(x_{\infty}\right)}$. We consider $q$ as in Lemma 4.1. In particular we know that $\left\|H_{k}(x, .)\right\|_{L^{q}\left(\Omega_{k}\right)} \leq C$ uniformly in $k$. By applying local elliptic estimates (cf. [1, Theorem 15.3]) combined with Sobolev embeddings in $\Omega_{k} \backslash \overline{B_{2 \delta}\left(x_{\infty}\right)}$ we find that

$$
\left\|H_{k}\left(x_{k}, .\right)\right\|_{L^{\infty}\left(\Omega_{k} \backslash \overline{B_{3 \delta}\left(x_{\infty}\right)}\right)} \leq C(q, \delta)
$$

uniformly in $k$. In particular, we would have

$$
\left|H_{k}\left(x_{k}, y_{k}\right)\right| \leq C(q, \delta) \quad \text { and } \quad\left|x_{k}-y_{k}\right|^{n-4}\left|H_{k}\left(x_{k}, y_{k}\right)\right| \leq C(q, \delta)
$$

independent of $k$. This contradicts our hypothesis (38).

Concluding the proof of Theorem 4, case $n>4$. In what follows we may work in one fixed coordinate domain $U_{i}$; for this reason we drop the index $i$. Let $\Phi_{k}: U \rightarrow \mathbb{R}^{n}$ be coordinate charts of $\Omega_{k}$ at $x_{\infty}$ as in Definition 1. We recall that

$$
\Phi_{k}\left(U \cap\left\{x_{1}<0\right\}\right)=\Phi_{k}(U) \cap \Omega_{k} \text { and } \Phi_{k}\left(U \cap\left\{x_{1}=0\right\}\right)=\Phi_{k}(U) \cap \partial \Omega_{k} .
$$

Without loss of generality we may assume that $\Phi_{k}(0)=x_{\infty}$ and $B_{\delta}\left(x_{\infty}\right) \subset \Phi_{k}(U)$.

We let $x_{k}=\Phi_{k}\left(x_{k}^{\prime}\right)$ and $y_{k}=\Phi_{k}\left(y_{k}^{\prime}\right)$. Therefore, (39) rewrites as

$$
\lim _{k \rightarrow+\infty} x_{k}^{\prime}=0 \text { and } \lim _{k \rightarrow+\infty} \frac{x_{k, 1}^{\prime}}{\left|x_{k}^{\prime}-y_{k}^{\prime}\right|}=0 .
$$

We define for $R$ large enough

$$
\tilde{H}_{k}(z)=\left|x_{k}^{\prime}-y_{k}^{\prime}\right|^{n-4} H_{k}\left(\Phi_{k}\left(x_{k}^{\prime}\right), \Phi_{k}\left(x_{k}^{\prime}+\left|x_{k}^{\prime}-y_{k}^{\prime}\right|\left(z-\rho_{k} \vec{e}_{1}\right)\right)\right)
$$

in $B_{R}(0) \cap\left\{x_{1}<0\right\}$, where $\rho_{k}:=\frac{x_{k, 1}^{\prime}}{\left|x_{k}^{\prime}-y_{k}^{\prime}\right|}$. We rewrite the biharmonic equation $\Delta^{2} H_{k}(x,)=$.0 complemented with Dirichlet boundary conditions as

$$
\Delta_{g_{k}}^{2} \tilde{H}_{k}=0 \text { in }\left(B_{R}(0) \cap\left\{z_{1}<0\right\}\right) \backslash\left\{\rho_{k} \vec{e}_{1}\right\}, \quad \tilde{H}_{k}=\partial_{1} \tilde{H}_{k}=0 \text { on }\left\{z_{1}=0\right\} .
$$

Here, $g_{k}(z)=\Phi_{k}^{*}(\mathcal{E})\left(x_{k}^{\prime}+\left|x_{k}^{\prime}-y_{k}^{\prime}\right|\left(z-\rho_{k} \vec{e}_{1}\right)\right), \mathcal{E}=\left(\delta_{i j}\right)$ the Euclidean metric, and $\Delta_{g_{k}}$ denotes the Laplace-Beltrami operator with respect to this rescaled and translated pull back of the Euclidean metric under $\Phi_{k}$. Then, for $\tau>0$ being chosen suitably small, it follows from elliptic estimates (see [1, Theorem 15.3]) and Sobolev embeddings that there exists $C(R, q, \tau)>0$ such that

$$
\left|\tilde{H}_{k}(z)\right| \leq C(R, q, \tau)\left\|\tilde{H}_{k}\right\|_{L^{q}\left(B_{R}(0) \backslash B_{\tau}(0)\right)}
$$


for all $z \in B_{R / 2}(0) \backslash B_{2 \tau}(0), z_{1} \leq 0$. In order to estimate the $L^{q}$-norm on the right-hand side we use (40) and obtain that

$$
\begin{aligned}
\int_{B_{R}(0) \cap\left\{\zeta_{1}<0\right\}}\left|\tilde{H}_{k}(\zeta)\right|^{q} d \zeta & \leq C\left|x_{k}^{\prime}-y_{k}^{\prime}\right|^{q(n-4)-n} \int_{\Omega_{k}}\left|H_{k}\left(x_{k}, y\right)\right|^{q} d y \\
& \leq C\left|x_{k}^{\prime}-y_{k}^{\prime}\right|^{q(n-4)-n} d\left(x_{k}, \partial \Omega_{k}\right)^{(4-n) q+n} \\
& \leq C\left(\frac{d\left(x_{k}, \partial \Omega_{k}\right)}{\left|x_{k}^{\prime}-y_{k}^{\prime}\right|}\right)^{n-q(n-4)} .
\end{aligned}
$$

Therefore, with (39), we get that $\lim _{k \rightarrow+\infty}\left\|\tilde{H}_{k}\right\|_{L^{q}\left(B_{R}(0) \backslash B_{\tau}(0)\right)}=0$, and (42) yields

$$
\lim _{k \rightarrow+\infty} \tilde{H}_{k}=0 \text { in } C^{0}\left(\left(B_{R / 2}(0) \backslash B_{2 \tau}(0)\right) \cap\left\{z_{1} \leq 0\right\}\right) .
$$

In particular, since $\lim _{k \rightarrow+\infty} \rho_{k}=0$, we have that

$$
\lim _{k \rightarrow+\infty} \tilde{H}_{k}\left(\frac{y_{k}^{\prime}-x_{k}^{\prime}}{\left|y_{k}^{\prime}-x_{k}^{\prime}\right|}+\rho_{k} \vec{e}_{1}\right)=0
$$

This limit rewrites as

$$
\lim _{k \rightarrow+\infty}\left|x_{k}-y_{k}\right|^{n-4}\left|H_{k}\left(x_{k}, y_{k}\right)\right|=0
$$

contradicting (38). The proof of Theorem $n>4$, is complete.

Proof of Theorem 4, case $n=4$. Here it is enough to prove (37) for $\nabla_{y}$, exploiting the symmetry of the Green's function. We argue by contradiction and as in the proof for $n>4$, we may assume that there exist two sequences $\left(x_{k}\right)_{k \in \mathbb{N}},\left(y_{k}\right)_{k \in \mathbb{N}}$ with $x_{k}, y_{k} \in \Omega_{k}$ such that $x_{k} \neq y_{k}$ and

$$
\lim _{k \rightarrow+\infty}\left|x_{k}-y_{k}\right|\left|\nabla_{y} H_{k}\left(x_{k}, y_{k}\right)\right|=+\infty
$$

After possibly passing to a subsequence, it follows from (10) that there exists $x_{\infty} \in$ $\partial \Omega$ such that

$$
\lim _{k \rightarrow+\infty} x_{k}=x_{\infty} \text { and } \lim _{k \rightarrow+\infty} \frac{d\left(x_{k}, \partial \Omega_{k}\right)}{\left|x_{k}-y_{k}\right|}=0 .
$$

Lemma 4.1 may be applied with some $q>4$. The analogue of Lemma 4.2 is proved in exactly the same way as above. Like above we now put for $R$ large enough

$$
\tilde{H}_{k}(z)=H_{k}\left(\Phi_{k}\left(x_{k}^{\prime}\right), \Phi_{k}\left(x_{k}^{\prime}+\left|x_{k}^{\prime}-y_{k}^{\prime}\right|\left(z-\rho_{k} \vec{e}_{1}\right)\right)\right)
$$

in $B_{R}(0) \cap\left\{z_{1}<0\right\}$, where $x_{k}=\Phi_{k}\left(x_{k}^{\prime}\right), y_{k}=\Phi_{k}\left(y_{k}^{\prime}\right), \rho_{k}:=\frac{x_{k, 1}^{\prime}}{\left|x_{k}^{\prime}-y_{k}^{\prime}\right|}$. As above we find for $\tau>0$ small enough that there exists $C(R, \tau, q)>0$ such that

$$
\left|\nabla \tilde{H}_{k}(z)\right| \leq C(R, q, \tau)\left\|\tilde{H}_{k}\right\|_{L^{q}\left(B_{R}(0) \backslash B_{\tau}(0)\right)}
$$

for all $z \in B_{R / 2}(0) \backslash B_{2 \tau}(0), z_{1} \leq 0$. Using (40) we obtain that

$$
\begin{aligned}
\int_{B_{R}(0) \cap\left\{\zeta_{1}<0\right\}}\left|\tilde{H}_{k}(\zeta)\right|^{q} d \zeta & \leq C\left|x_{k}^{\prime}-y_{k}^{\prime}\right|^{-4} \int_{\Omega_{k}}\left|H_{k}\left(x_{k}, y\right)\right|^{q} d y \\
& \leq C\left(\frac{d\left(x_{k}, \partial \Omega_{k}\right)}{\left|x_{k}^{\prime}-y_{k}^{\prime}\right|}\right)^{4} .
\end{aligned}
$$

In the same way as in the generic case $n>4$, this yields first that

$$
\lim _{k \rightarrow+\infty} \nabla \tilde{H}_{k}=0 \text { in } C^{0}\left(\left(B_{R / 2}(0) \backslash B_{2 \tau}(0)\right) \cap\left\{z_{1} \leq 0\right\}\right)
$$


and back in the original coordinates

$$
\lim _{k \rightarrow \infty}\left|x_{k}-y_{k}\right|\left|\nabla_{y} H_{k}\left(x_{k}, y_{k}\right)\right|=0 .
$$

So, we achieve a contradiction also if $n=4$. This proves (37). Integrating (37), we get (36). The proof of Theorem 4 is complete.

\section{Convergence of the Green's functions}

As before, we consider a family of bounded regular domains $\left(\Omega_{k}\right)$ being a $C^{4, \alpha_{-}}$ smooth perturbation of a fixed bounded $C^{4, \alpha}$-smooth domain $\Omega$ according to Definition 1. We consider the operators $\Delta^{2}+a_{k}$ in $\Omega_{k}$ and assume that

$$
\begin{gathered}
\exists U_{0} \supset \overline{\Omega_{k}}: a_{k} \in C^{0, \alpha}\left(U_{0}\right) ; \\
\exists a_{\infty} \in C^{0, \alpha}\left(U_{0}\right): \lim _{k \rightarrow \infty} a_{k}=a_{\infty} \text { in } C_{l o c}^{0, \alpha}\left(U_{0}\right) .
\end{gathered}
$$

As before, we denote by $G_{k}$ the Green's functions corresponding to $\Delta^{2}+a_{k}$ in $\Omega_{k}$ and by $G$ the Green's functions corresponding to $\Delta^{2}+a_{\infty}$ in $\Omega$ and show the following convergence result. As for the diffeomorphisms $\Phi_{k, i}, \Phi_{i}$ we refer to Definition 1

Proposition 5.1. Let $x_{k} \in \Omega_{k}$ and assume that $\lim _{k \rightarrow \infty} x_{k}=x_{\infty} \in \Omega$. Then, we have:

$$
\begin{aligned}
G_{k}\left(x_{k}, .\right) & \rightarrow G\left(x_{\infty}, .\right) \text { in } C_{l o c}^{4}\left(\Omega \backslash\left\{x_{\infty}\right\}\right), \\
G_{k}\left(x_{k}, .\right) & \rightarrow G\left(x_{\infty}, .\right) \text { in } L^{1}\left(\mathbb{R}^{n}\right), \\
G_{k}\left(x_{k}, .\right) \circ \Phi_{k, i} & \rightarrow G\left(x_{\infty}, .\right) \circ \Phi_{i} \text { in } C_{l o c}^{4}\left(U_{i} \cap\left\{z_{1} \leq 0\right\} \backslash\left\{\Phi_{i}^{-1}\left(x_{\infty}\right)\right\}\right) .
\end{aligned}
$$

If $n=3$ we have in addition that

$$
G_{k}(., .) \rightarrow G(., .) \text { in } C_{l o c}^{0}(\Omega \times \Omega) .
$$

Proof. According to Theorem 4 and Proposition 3.2 we know that

$$
\begin{aligned}
& \left|H_{k}(x, y)\right| \leq C \cdot \begin{cases}|x-y|^{4-n}, & \text { if } n>4, \\
(1+|\log | x-y||), & \text { if } n=4, \\
1, & \text { if } n=3 ;\end{cases} \\
& \left|G_{k}(x, y)\right| \leq C \cdot \begin{cases}|x-y|^{4-n}, & \text { if } n>4, \\
(1+|\log | x-y||), & \text { if } n=4, \\
1, & \text { if } n=3 ;\end{cases}
\end{aligned}
$$

uniformly in $k$. This shows that in particular

$$
\left\|G_{k}(x, .)\right\|_{L^{1}\left(\Omega_{k}\right)} \leq C \text { uniformly in } k .
$$

Moreover, since $x_{k} \rightarrow x_{\infty}$, we may assume that all $x_{k}$ are in a small neighbourhood around $x_{\infty}$. Refering to the construction in the proof of Proposition 3.1 we see that the $u_{k, x_{k}}$ arising there are uniformly bounded in $C^{4, \alpha}\left(\overline{\Omega_{k}}\right)$. After selecting a suitable subsequence we see that for each $\Omega_{0} \subset \subset \Omega$ one has $G_{k}\left(x_{k},.\right) \rightarrow \varphi$ in $C_{l o c}^{4}\left(\overline{\Omega_{0}} \backslash\left\{x_{\infty}\right\}\right)$ and $G_{k}\left(x_{k},.\right) \circ \Phi_{k, i} \rightarrow \varphi \circ \Phi_{i}$ in $C_{l o c}^{4}\left(U_{i} \cap\left\{z_{1} \leq 0\right\} \backslash\left\{\Phi_{i}^{-1}\left(x_{\infty}\right)\right\}\right)$ with a suitable $\varphi \in C^{4, \alpha}\left(\bar{\Omega} \backslash\left\{x_{\infty}\right\}\right)$. Thanks to this compactness and the fact that in any case the limit is the uniquely determined Green's function, we have convergence on the whole sequence towards $G\left(x_{\infty},.\right)$. 
Finally, since we have pointwise convergence, (45) allows for applying Vitali's convergence theorem to show that

$$
G_{k}\left(x_{k}, .\right) \rightarrow G\left(x_{\infty}, .\right) \text { in } L^{1}\left(\mathbb{R}^{n}\right) .
$$

The statement concerning $C_{l o c}^{0}(\Omega \times \Omega)$-convergence in $n=3$ follows from $\left|\nabla G_{k}(.,).\right| \leq$ $C$, cf. (10).

In order to prove Lemma 6.4 below we also need a convergence result simultaneous in both variables.

Proposition 5.2. We have that

$$
\begin{array}{r}
G_{k}(., .) \circ\left(\Phi_{k, i} \times \Phi_{k, j}\right) \rightarrow G(., .) \circ\left(\Phi_{i} \times \Phi_{j}\right) \\
\text { in } C_{l o c}^{4}\left(\left(U_{i} \cap\left\{x_{1} \leq 0\right\}\right) \times\left(U_{j} \cap\left\{x_{1} \leq 0\right\}\right) \backslash D_{i j}\right), \text { where } \\
D_{i j}=\left\{(x, y) \in U_{i} \times U_{j}: \Phi_{i}(x)=\Phi_{j}(y)\right\} .
\end{array}
$$

Proof. We combine the ideas of the proofs of Propositions 5.1 and 3.3. One should observe that Theorem 4 and Proposition 3.2 guarantee uniform $L^{1}$-bounds for $H_{k}$ and $G_{k}$ as in the proof of Proposition 5.1 .

\section{The limit of the zeros of the Green's functions}

We keep the notations of the previous sections. In order to prove Theorem 3, we assume that for every $k$, there exist

$$
x_{k}, y_{k} \in \Omega_{k}, x_{k} \neq y_{k}: \quad G_{k}\left(x_{k}, y_{k}\right)=0 .
$$

After passing to subsequences there exist $x_{\infty}=\lim _{k \rightarrow \infty} x_{k}, y_{\infty}=\lim _{k \rightarrow \infty} y_{k}$. Using Definition 1, one sees that $x_{\infty}, y_{\infty} \in \bar{\Omega}$.

As for the location of these limit points, we distinguish several cases.

6.1. Both points in the interior. Here, we consider the case that $x_{\infty}, y_{\infty} \in \Omega$. Once it is shown that $x_{\infty} \neq y_{\infty}$ we conclude directly from Proposition 5.1 that

$$
G\left(x_{\infty}, y_{\infty}\right)=0
$$

So, we are left with proving:

Lemma 6.1. $x_{\infty} \neq y_{\infty}$.

Proof. Assume by contradiction that $x_{\infty}=y_{\infty}$. We consider first the case $n>4$ and here, the rescaled Green's function:

$$
\tilde{G}_{k}(z):=\left|x_{k}-y_{k}\right|^{n-4} G_{k}\left(x_{k}, x_{k}+\left|x_{k}-y_{k}\right| z\right) .
$$

Let $\varepsilon>0$ be such that $\overline{B_{2 \varepsilon}\left(x_{\infty}\right)} \subset \Omega \cap \Omega_{k}$ for all $k$. Then, for $k$ large enough, $\left|x_{k}-x_{\infty}\right|<\varepsilon$ and $\tilde{G}_{k}(z)$ is certainly defined for $|z|<\frac{\varepsilon}{\left|x_{k}-y_{k}\right|}$, where one has by Theorem 4 and Proposition 3.2 that

$$
\left|\tilde{G}_{k}(z)\right| \leq C|z|^{4-n}
$$

uniformly in $k$. Because the $\tilde{G}_{k}$ are defined on a sequence of sets which exhausts the whole $\mathbb{R}^{n}$ we may discuss how to pass to a limit locally in $\mathbb{R}^{n}$. Since

$$
\Delta^{2} \tilde{G}_{k}+\left|x_{k}-y_{k}\right|^{4} a_{k}\left(x_{k}+\left|x_{k}-y_{k}\right| z\right) \tilde{G}_{k}=0 \text { on } \overline{B_{\varepsilon /\left|x_{k}-y_{k}\right|}(0)} \backslash\{0\},
$$

by elliptic Schauder theory we may assume that after possibly passing to a subsequence that

$$
\tilde{G}_{k} \rightarrow \tilde{G} \text { in } C_{l o c}^{4}\left(\mathbb{R}^{n} \backslash\{0\}\right) \text {, where }|\tilde{G}(z)| \leq C|z|^{4-n} \text {. }
$$


Moreover,

$$
\Delta^{2} \tilde{G}=0 \text { in } \mathbb{R}^{n} \backslash\{0\} .
$$

In order to compute the differential equation satisfied by $\tilde{G}$ near $z=0$, let $\varphi \in$ $C_{c}^{\infty}\left(\mathbb{R}^{n}\right)$ with supp $\varphi \subset B_{R}(0)$ and define for $k$ large enough

$$
\begin{aligned}
& \Omega_{k} \ni x \mapsto \varphi_{k}(x):=\varphi\left(\frac{x-x_{k}}{\left|x_{k}-y_{k}\right|}\right), \quad \varphi_{k} \in C_{c}^{\infty}\left(\Omega_{k}\right) . \\
\varphi(0)= & \varphi_{k}\left(x_{k}\right)=\int_{\Omega_{k}} G_{k}\left(x_{k}, y\right)\left(\Delta^{2} \varphi_{k}+a_{k} \varphi_{k}\right) d y \\
= & \int_{B_{\left|x_{k}-y_{k}\right| R}\left(x_{k}\right)} G_{k}\left(x_{k}, y\right)\left|x_{k}-y_{k}\right|^{-4} \\
= & \left.\int_{B_{R}(0)} \cdot\left(\Delta^{2} \varphi\right)\left(\frac{y-x_{k}}{\left|x_{k}-y_{k}\right|}\right)+\left|x_{k}-y_{k}\right|^{4} a_{k}(y) \varphi\left(\frac{y-x_{k}}{\left|x_{k}-y_{k}\right|}\right)\right) d y \\
= & \int_{\mathbb{R}^{n}}\left(\Delta^{2} \varphi(z)+\left|x_{k}-x_{k}+\right| x_{k}-\left.y_{k}\right|^{4} a_{k}\left(x_{k}+\left|x_{k}-y_{k}\right| z\right) \varphi(z)\right) d z
\end{aligned}
$$

We put $\gamma_{n}=\frac{1}{2(n-4)(n-2) n e_{n}}$ and obtain, letting $k \rightarrow \infty$ :

$$
\int_{\mathbb{R}^{n}} \tilde{G}(z) \Delta^{2} \varphi(z) d z=\varphi(0)=\int_{\mathbb{R}^{n}} \gamma_{n}|z|^{4-n} \Delta^{2} \varphi(z) d z .
$$

This shows that we have in the sense of distributions that

$$
\Delta^{2}\left(\tilde{G}(z)-\gamma_{n}|z|^{4-n}\right)=0 \text { in } \mathbb{R}^{n}
$$

Hence,

$$
\tilde{G}(z)=\gamma_{n}|z|^{4-n}+\psi(z), \quad \psi \in C^{\infty}\left(\mathbb{R}^{n}\right), \quad \Delta^{2} \psi=0 .
$$

Thanks to (50) we know further that

$$
|\psi(z)| \leq C(1+|z|)^{4-n} .
$$

Also for entire bounded biharmonic (even more generally polyharmonic) functions, Liouville's theorem holds true, i.e. these are constant, see [25, p. 19]. Hence $\psi(z) \equiv 0$ showing that

$$
\tilde{G}(z)=\gamma_{n}|z|^{4-n}, \quad z \in \mathbb{R}^{n} \backslash\{0\} .
$$

On the other hand we have according to the choice (47) of $x_{k}, y_{k}$ and the definition (49) of $\tilde{G}_{k}$ that

$$
\tilde{G}_{k}\left(\frac{y_{k}-x_{k}}{\left|x_{k}-y_{k}\right|}\right)=\left|x_{k}-y_{k}\right|^{n-4} G_{k}\left(x_{k}, y_{k}\right)=0 .
$$

Hence there exists at least one point $\zeta \in \mathbb{R}^{n}$ with

$$
|\zeta|=1 \text { and } 0=\tilde{G}(\zeta)=\gamma_{n}|\zeta|^{4-n},
$$

which is false. This proves the statement for the case $n>4$. One should observe that when looking just at the biharmonic operator, a proof for the previous lemma would directly follow from the local positivity results in general domains, which are proved in [16]. This observation will be useful in what follows. 
Let us now consider the case $n=4$. Since $x_{\infty} \in \Omega$, according to [16], there exists (a small) $\delta_{1}>0$ such that for all $k$ and all $x, y \in \Omega_{k}$ we have that

$$
x, y \in B_{\delta_{1}}\left(x_{\infty}\right) \quad \Rightarrow \quad H_{k}(x, y) \geq-\frac{1}{c_{3}} \log |x-y| .
$$

We estimate the difference between $G_{k}$ and $H_{k}$. For arbitrary but fixed $x \in \Omega$, we have that with respect to the $y$-variable, $\left(H_{k}-G_{k}\right)(x,$.$) solves the following$ Dirichlet problem:

$$
\begin{cases}\Delta_{y}^{2}\left(H_{k}-G_{k}\right)(x, y)+a_{k}(y)\left(H_{k}-G_{k}\right)(x, y)=a_{k}(y) H_{k}(x, y), & y \in \Omega_{k} \\ \left(H_{k}-G_{k}\right)(x, y)=\frac{\partial}{\partial \nu_{y}}\left(H_{k}-G_{k}\right)(x, y)=0, & y \in \partial \Omega_{k} .\end{cases}
$$

According to Theorem 4, we have that $\left\|a_{k}(.) H_{k}(x, .)\right\|_{L^{2}\left(\Omega_{k}\right)} \leq c_{4}$ uniformly in $k$ and $x$. Since $\Delta^{2}+a_{k}$ is assumed to be uniformly coercive, elliptic estimates from [1] show that

$$
\left\|\left(H_{k}-G_{k}\right)(x, .)\right\|_{L^{\infty}\left(\Omega_{k}\right)} \leq C\left\|\left(H_{k}-G_{k}\right)(x, .)\right\|_{W^{4,2}\left(\Omega_{k}\right)} \leq c_{5},
$$

uniformly in $x$ and $k$. Together with (52), this gives that there exist a $\delta_{2}>0$ and a constant $c_{6}>0$ such that

$$
x, y \in B_{\delta_{2}}\left(x_{\infty}\right) \quad \Rightarrow \quad G_{k}(x, y) \geq-\frac{1}{c_{6}} \log |x-y| .
$$

This proves the claim also for $n=4$, since by (53), it is impossible that $G_{k}\left(x_{k}, y_{k}\right)=$ 0 , where $x_{k}, y_{k} \rightarrow x_{\infty} \in \Omega$.

Finally, we consider $n=3$. Since here, according to Proposition 5.1, also $G_{k} \rightarrow G$ in $C_{l o c}^{0}(\Omega \times \Omega)$ we have by assumption that $G\left(x_{\infty}, x_{\infty}\right)=0$. On the other hand, testing the boundary value problem for $G\left(x_{\infty}\right.$, . ) with $G\left(x_{\infty}\right.$, . ) itself yields by virtue of the uniform coercivity that

$$
G\left(x_{\infty}, x_{\infty}\right) \geq \lambda \int_{\Omega} G\left(x_{\infty}, y\right)^{2} d y>0 .
$$

We obtain a contradiction also in the case $n=3$. So, the proof of Lemma 6.1 is complete.

6.2. One point in the interior, one point on the boundary. After possibly interchanging $x_{\infty}$ and $y_{\infty}$ we may consider the case that $x_{\infty} \in \Omega, y_{\infty} \in \partial \Omega$.

\section{Lemma 6.2.}

$$
\Delta_{y} G\left(x_{\infty}, y_{\infty}\right)=0
$$

Proof. We may fix a neighbourhood $B_{\delta}\left(p_{i}\right)$ such that $y_{\infty} \in \partial \Omega \cap B_{\delta}\left(p_{i}\right)$ so that for $k$ large enough $y_{k} \in \Omega_{k} \cap B_{\delta}\left(p_{i}\right)$. We denote $y_{k}^{\prime}:=\Phi_{k, i}^{-1}\left(y_{k}\right), y_{\infty}^{\prime}:=\Phi_{i}^{-1}\left(y_{\infty}\right)$ and observe that $\left(y_{k}^{\prime}\right)_{1}<0,\left(y_{\infty}^{\prime}\right)_{1}=0, y_{k}^{\prime} \rightarrow y_{\infty}^{\prime}$ in $U_{i}$. we recall the notation $y_{k}^{\prime}=\left(y_{k, 1}^{\prime}, \overline{y_{k}^{\prime}}\right)$. Writing

$$
\tilde{G}_{k, i}:=G_{k}\left(x_{k}, .\right) \circ \Phi_{k, i}, \quad \tilde{G}_{i}:=G_{k}\left(x_{\infty}, .\right) \circ \Phi_{i}
$$

we see by means of Taylor's expansion that with suitable $\theta_{k} \in(0,1)$ :

$$
\begin{aligned}
0 & =G_{k}\left(x_{k}, y_{k}\right)=\tilde{G}_{k, i}\left(y_{k}^{\prime}\right) \\
& =\tilde{G}_{k, i}\left(0, \bar{y}_{k}^{\prime}\right)+\partial_{1} \tilde{G}_{k, i}\left(0, \bar{y}_{k}^{\prime}\right) y_{k, 1}^{\prime}+\frac{1}{2} \partial_{11} \tilde{G}_{k, i}\left(\theta_{k} y_{k, 1}^{\prime}, \overline{y_{k}^{\prime}}\right)\left(y_{k, 1}^{\prime}\right)^{2} \\
& =\frac{1}{2} \partial_{11} \tilde{G}_{k, i}\left(\theta_{k} y_{k, 1}^{\prime}, \overline{y_{k}^{\prime}}\right)\left(y_{k, 1}^{\prime}\right)^{2}
\end{aligned}
$$


due to the boundary conditions on $G_{k}$. According to Proposition 5.1 this yields $\partial_{11} \tilde{G}_{i}\left(y_{\infty}^{\prime}\right)=0$. Since $\left.G_{k}\left(x_{k},.\right)\right|_{\partial \Omega}=\left.\frac{\partial}{\partial \nu} G_{k}\left(x_{k},.\right)\right|_{\partial \Omega}=0$, we obtain back in the original coordinates that $\Delta_{y} G\left(x_{\infty}, y_{\infty}\right)=0$ as stated.

6.3. Both points on the boundary. So, here we have to consider the case that both $x_{\infty} \in \partial \Omega$ and $y_{\infty} \in \partial \Omega$. The most delicate part will be to prove that both points have to be distinct:

Lemma 6.3. $x_{\infty} \neq y_{\infty}$.

The proof is rather technical and will be postponed to Subsection 6.4 Assuming now Lemma 6.3 being proved it is not too difficult that in this case an additional zero of the Green's function can be observed on the boundary:

Lemma 6.4. $\Delta_{x} \Delta_{y} G\left(x_{\infty}, y_{\infty}\right)=0$.

Proof. According to Proposition 3.3 we have that $G \in C^{4, \alpha}$ in a neighbourhood of $\left(x_{\infty}, y_{\infty}\right)$. This proof is similar to that of Lemma 6.2. We fix neighbourhoods such that $x_{\infty} \in B_{\delta}\left(p_{i}\right), y_{\infty} \in B_{\delta}\left(p_{j}\right)$; without loss of generality we may assume that $B_{\delta}\left(p_{i}\right) \cap B_{\delta}\left(p_{j}\right)=\emptyset$. Moreover we may assume that $\forall k: \quad x_{k} \in B_{\delta}\left(p_{i}\right), y_{k} \in B_{\delta}\left(p_{j}\right)$. To work in local charts we define

$$
x_{k}^{\prime}:=\Phi_{k, i}^{-1}\left(x_{k}\right), \quad x_{\infty}^{\prime}:=\Phi_{i}^{-1}\left(x_{\infty}\right), \quad y_{k}^{\prime}:=\Phi_{k, j}^{-1}\left(y_{k}\right), \quad y_{\infty}^{\prime}:=\Phi_{j}^{-1}\left(y_{\infty}\right) .
$$

Hence we have

Defining

$$
\begin{array}{ll}
x_{k}^{\prime} \in U_{i} \cap\left\{x_{1}<0\right\}, & x_{k}^{\prime} \rightarrow x_{\infty}^{\prime} \in U_{i} \cap\left\{x_{1}=0\right\}, \\
y_{k}^{\prime} \in U_{j} \cap\left\{y_{1}<0\right\}, & y_{k}^{\prime} \rightarrow y_{\infty}^{\prime} \in U_{j} \cap\left\{y_{1}=0\right\} .
\end{array}
$$

$$
\begin{gathered}
\tilde{G}_{k}: U_{i} \cap\left\{x_{1} \leq 0\right\} \times U_{j} \cap\left\{y_{1} \leq 0\right\} \rightarrow \mathbb{R}, \tilde{G}_{k}\left(x^{\prime}, y^{\prime}\right):=G_{k}\left(\Phi_{k, i}\left(x^{\prime}\right), \Phi_{k, j}\left(y^{\prime}\right)\right) ; \\
\tilde{G}: U_{i} \cap\left\{x_{1} \leq 0\right\} \times U_{j} \cap\left\{y_{1} \leq 0\right\} \rightarrow \mathbb{R}, \tilde{G}\left(x^{\prime}, y^{\prime}\right):=G_{k}\left(\Phi_{i}\left(x^{\prime}\right), \Phi_{j}\left(y^{\prime}\right)\right)
\end{gathered}
$$

we see that by assumption

$$
0=G_{k}\left(x_{k}, y_{k}\right)=\tilde{G}_{k}\left(x_{k}^{\prime}, y_{k}^{\prime}\right) .
$$

Taylor's expansion with respect to $y^{\prime}$ and exploiting the boundary conditions for $\tilde{G}_{k}$ with respect to $y^{\prime}$ shows that for each $k$ there exists a suitable $\theta_{k} \in(0,1)$ such that

$$
\partial_{y_{1}}^{2} \tilde{G}_{k}\left(x_{k, 1}^{\prime}, \overline{x_{k}^{\prime}}, \theta_{k} y_{k, 1}^{\prime}, \overline{y_{k}^{\prime}}\right)=0
$$

Now, we use Taylor's expansion for this expression with respect to $x^{\prime}$ and obtain with suitable $\tau_{k} \in(0,1)$ :

$$
\begin{aligned}
& 0=\partial_{y_{1}}^{2} \tilde{G}_{k}\left(x_{k, 1}^{\prime}, \bar{x}_{k}^{\prime}, \theta_{k} y_{k, 1}^{\prime}, \overline{y_{k}^{\prime}}\right) \\
& =\partial_{y_{1}}^{2} \tilde{G}_{k}\left(0, \overline{x_{k}^{\prime}}, \theta_{k} y_{k, 1}^{\prime}, \overline{y_{k}^{\prime}}\right)+\partial_{x_{1}} \partial_{y_{1}}^{2} \tilde{G}_{k}\left(0, \overline{x_{k}^{\prime}}, \theta_{k} y_{k, 1}^{\prime}, \bar{y}_{k}^{\prime}\right) x_{k, 1}^{\prime} \\
& +\frac{1}{2} \partial_{x_{1}}^{2} \partial_{y_{1}}^{2} \tilde{G}_{k}\left(\tau_{k} x_{k, 1}^{\prime}, \bar{x}_{k}^{\prime}, \theta_{k} y_{k, 1}^{\prime}, \bar{y}_{k}^{\prime}\right)\left(x_{k, 1}^{\prime}\right)^{2} \\
& =\frac{1}{2} \partial_{x_{1}}^{2} \partial_{y_{1}}^{2} \tilde{G}_{k}\left(\tau_{k} x_{k, 1}^{\prime}, \overline{x_{k}^{\prime}}, \theta_{k} y_{k, 1}^{\prime}, \overline{y_{k}^{\prime}}\right)\left(x_{k, 1}^{\prime}\right)^{2}
\end{aligned}
$$

so that

$$
\partial_{x_{1}}^{2} \partial_{y_{1}}^{2} \tilde{G}_{k}\left(\tau_{k} x_{k, 1}^{\prime}, \bar{x}_{k}^{\prime}, \theta_{k} y_{k, 1}^{\prime}, \overline{y_{k}^{\prime}}\right)=0
$$

Since by Proposition 5.2 we have $C^{4}$ convergence of $\tilde{G}_{k}$ to $\tilde{G}$ it follows that

$$
\partial_{x_{1}}^{2} \partial_{y_{1}}^{2} \tilde{G}\left(x_{\infty}^{\prime}, y_{\infty}^{\prime}\right)=0
$$


Taking into account the boundary conditions of $G$ and of $\tilde{G}$, back in the original variables we see that

$$
\Delta_{x} \Delta_{y} G\left(x_{\infty}, y_{\infty}\right)=0
$$

thereby proving the claim.

6.4. Proof of Lemma 6.3. We assume by contradiction that $\lim _{k \rightarrow \infty} x_{k}=x_{\infty}=$ $y_{\infty}=\lim _{k \rightarrow \infty} y_{k}$. We choose a neighbourhood $B_{\delta}\left(p_{i}\right) \ni x_{\infty}$ and may assume that $\forall k: x_{k}, y_{k} \in B_{\delta}\left(p_{i}\right) \cap \Omega_{k}$. As before we introduce

$$
x_{k}^{\prime}:=\Phi_{k, i}^{-1}\left(x_{k}\right), \quad y_{k}^{\prime}:=\Phi_{k, i}^{-1}\left(y_{k}\right), \quad x_{\infty}^{\prime}:=\Phi_{i}^{-1}\left(x_{\infty}\right)
$$

so that we have

$$
x_{k}^{\prime}, y_{k}^{\prime} \in U_{i} \cap\left\{x_{1}<0\right\}, \quad x_{k}^{\prime} \rightarrow x_{\infty}^{\prime}, y_{k}^{\prime} \rightarrow x_{\infty}^{\prime} \in U_{i} \cap\left\{x_{1}=0\right\} .
$$

We distinguish two further cases according to whether the distance between $x_{k}$ and $y_{k}$ converges faster to 0 than the distance of these points to the boundary or vice verca.

First case: $\left|x_{k}-y_{k}\right|=o\left(\max \left(d\left(x_{k}, \partial \Omega_{k}\right), d\left(y_{k}, \partial \Omega_{k}\right)\right)\right)$. After possibly interchanging $x_{k}$ and $y_{k}$ and passing to a subsequence we may assume that

$$
\left|x_{k}-y_{k}\right|=o\left(d\left(x_{k}, \partial \Omega_{k}\right)\right) \text {. }
$$

This case is much simpler than the second case below and quite similar to the case where both points converge in the interior treated in Subsection 6.1 Like there we treat the case $n>4$ first. In this case, we consider the rescaled Green's functions:

$$
\tilde{G}_{k}(z):=\left|x_{k}-y_{k}\right|^{n-4} G_{k}\left(x_{k}, x_{k}+\left|x_{k}-y_{k}\right| z\right) .
$$

These are is certainly defined for $|z|<\frac{d\left(x_{k}, \partial \Omega_{k}\right)}{\left|x_{k}-y_{k}\right|}$, which converges to $\infty$ as $k \rightarrow \infty$. For this reason, we may now directly copy the reasoning of Subsection 6.1 and obtain that

$$
\tilde{G}_{k} \rightarrow \tilde{G} \text { in } C_{l o c}^{4}\left(\mathbb{R}^{n} \backslash\{0\}\right) \text { with } \tilde{G}(z)=\gamma_{n}|z|^{4-n} .
$$

One should observe that also here the property of the Green's functions to be uniformly bounded by $C|x-y|^{4-n}$ is used. According to the choice (47) of $x_{k}, y_{k}$ and the definition of $\tilde{G}_{k}$ we have that

$$
\tilde{G}_{k}\left(\frac{y_{k}-x_{k}}{\left|x_{k}-y_{k}\right|}\right)=\left|x_{k}-y_{k}\right|^{n-4} G_{k}\left(x_{k}, y_{k}\right)=0 .
$$

Hence there exists at least one point $\zeta \in \mathbb{R}^{n}$ with

$$
|\zeta|=1 \text { and } 0=\tilde{G}(\zeta)=\gamma_{n}|\zeta|^{4-n}
$$

which is false.

We now treat the case $n=4$ and proceed similarly as in the proof of Lemma 6.1 Rescaling the result of [16] shows the existence of $\delta>0, c_{3}>0$ such that for $x, y \in \Omega_{k}$ with $|x-y| \leq \delta d\left(x, \partial \Omega_{k}\right)$, one has (uniformly in $k$ ) that

$$
H_{k}(x, y) \geq-\frac{1}{c_{3}} \log \frac{|x-y|}{d\left(x, \partial \Omega_{k}\right)} .
$$

As it was shown in the proof of Lemma 6.1, $G_{k}-H_{k}$ is bounded uniformly in $k$. Hence, there exists a constant $c_{4}$ such that for $x, y \in \Omega_{k}$ we have

$$
|x-y| \leq \delta \mathrm{d}\left(x, \partial \Omega_{k}\right) \quad \Rightarrow \quad G_{k}(x, y) \geq-\frac{1}{c_{3}} \log \frac{|x-y|}{d\left(x, \partial \Omega_{k}\right)}-c_{4} .
$$


Since $\left|x_{k}-y_{k}\right|=o\left(d\left(x_{k}, \partial \Omega_{k}\right)\right)$ we obtain

$$
0=G_{k}\left(x_{k}, y_{k}\right) \rightarrow \infty \quad(k \rightarrow \infty) .
$$

This is again false and proves the claim for $n=4$.

Finally we discuss the case $n=3$. Rescaling the result of Nehari 24 shows the existence of $\delta>0, \varepsilon>0$ such that for $x, y \in \Omega_{k}$ with $|x-y| \leq \delta d\left(x, \partial \Omega_{k}\right)$, one has (uniformly in $k$ ) that

$$
H_{k}(x, y) \geq \varepsilon d\left(x, \partial \Omega_{k}\right) .
$$

Making use of elliptic theory as in the proof of Lemma 6.1 and exploiting the fact that $n=3$ yields that $\left\|\left(G_{k}\left(., y_{k}\right)-H_{k}\left(., y_{k}\right)\right)\right\|_{C^{2}\left(\overline{\Omega_{k}}\right)} \leq c_{5}$ uniformly in $k$. Since $\left|x_{k}-y_{k}\right| \leq \delta d\left(x_{k}, \partial \Omega_{k}\right)$ for $k$ large enough we conclude that

$$
0=G_{k}\left(x_{k}, y_{k}\right) \geq \varepsilon d\left(x_{k}, \partial \Omega_{k}\right)-c_{6} d\left(x_{k}, \partial \Omega_{k}\right)^{2},
$$

which becomes again false for $k \rightarrow \infty$.

Second case: $\left|x_{k}-y_{k}\right| \neq o\left(\max \left(d\left(x_{k}, \partial \Omega_{k}\right), d\left(y_{k}, \partial \Omega_{k}\right)\right)\right.$. After selecting a subsequence we may assume that there is $\tau>0$ such that

$$
\left|x_{k}-y_{k}\right| \geq \tau d\left(x_{k}, \partial \Omega_{k}\right) \text { and }\left|x_{k}-y_{k}\right| \geq \tau d\left(y_{k}, \partial \Omega_{k}\right) .
$$

We define

$$
\rho_{k}:=\frac{\left(x_{k}^{\prime}\right)_{1}}{\left|x_{k}^{\prime}-y_{k}^{\prime}\right|}<0 \text { and } O(1)
$$

and after selecting a further subsequence we may assume that

$$
\lim _{k \rightarrow \infty} \rho_{k}=: \rho \leq 0 .
$$

Again, we will introduce a rescaled family of Green's functions. For any $R>0$ and $z, \zeta \in B_{R} \cap \mathbb{R}_{-}^{n}$,

$$
\begin{array}{r}
\tilde{G}_{k}(z, \zeta):=\left|x_{k}^{\prime}-y_{k}^{\prime}\right|^{n-4} G_{k}\left(\Phi_{k, i}\left(x_{k}^{\prime}+\left|x_{k}^{\prime}-y_{k}^{\prime}\right|\left(z-\rho_{k} \vec{e}_{1}\right)\right),\right. \\
\left.\Phi_{k, i}\left(x_{k}^{\prime}+\left|x_{k}^{\prime}-y_{k}^{\prime}\right|\left(\zeta-\rho_{k} \vec{e}_{1}\right)\right)\right) .
\end{array}
$$

Moreover, $\tilde{G}_{k}(z, \cdot)=\partial_{\zeta_{1}} \tilde{G}_{k}(z, \cdot)=0$ on $B_{R}(0) \cap \partial \mathbb{R}_{-}^{n}$. According to (36) and Proposition 3.2 we see that uniformly in $k, z$ and $\zeta$

$$
\left|\tilde{G}_{k}(z, \zeta)\right| \leq C|z-\zeta|^{4-n}, \quad \text { provided that } n>4 .
$$

If $n=3,4$ we conclude first that

$$
\left|\nabla \tilde{G}_{k}(z, \zeta)\right| \leq C \cdot \begin{cases}|z-\zeta|^{-1}, & \text { if } n=4, \\ 1, & \text { if } n=3 .\end{cases}
$$

Upon integration we obtain that

$$
\left|\tilde{G}_{k}(z, \zeta)\right| \leq C \cdot \begin{cases}(1+|\log | z-\zeta||+\log (1+|z|)+\log (1+|\zeta|)), & \text { if } n=4, \\ (1+|z|+|\zeta|), & \text { if } n=3 .\end{cases}
$$

The points $x_{k}$ and $y_{k}$ were chosen such that $G_{k}\left(x_{k}, y_{k}\right)=0$, which reads in new coordinates

$$
\tilde{G}_{k}\left(\rho_{k} \vec{e}_{1}, \frac{y_{k}^{\prime}-x_{k}^{\prime}}{\left|x_{k}^{\prime}-y_{k}^{\prime}\right|}+\rho_{k} \vec{e}_{1}\right)=0
$$


In order to formulate the differential equation satisfied by $\tilde{G}_{k}$, we denote by $\mathcal{E}=$ $\left(\delta_{i j}\right)$ the Euclidean metric and

$$
g_{k, i}(z):=\Phi_{k, i}^{*}(\mathcal{E})\left(x_{k}^{\prime}+\left|x_{k}^{\prime}-y_{k}^{\prime}\right|\left(z-\rho_{k} \vec{e}_{1}\right)\right)
$$

its translated and rescaled pullback with respect to the coordinate charts $\Phi_{k, i}$. Moreover, we introduce its limit, the constant metric

$$
g_{\infty, i}:=\Phi_{i}^{*}(\mathcal{E})\left(x_{\infty}\right)
$$

First, we keep $z \in \mathbb{R}_{-}^{n}$ fixed and consider $\tilde{G}_{k}(z,)=.: \tilde{G}_{k, z}($.$) as function in the$ second variable. For $\zeta \in B_{R}(0) \cap \mathbb{R}_{-}^{n} \backslash\{z\}$ we have that for $k$ large enough, the following boundary value problem is satisfied:

$$
\begin{cases}\Delta_{g_{k, i}, \zeta}^{2} \tilde{G}_{k}(z, \zeta) & \\ \quad+\left|x_{k}^{\prime}-y_{k}^{\prime}\right|^{4}\left(a_{k} \circ \Phi_{k, i}\right) & \left(x_{k}^{\prime}+\left|x_{k}^{\prime}-y_{k}^{\prime}\right|\left(\zeta-\rho_{k} \vec{e}_{1}\right)\right) \tilde{G}_{k}(z, \zeta)=0 \\ & \text { for } \zeta_{1}<0, \zeta \neq z, \\ \tilde{G}_{k}(z, \zeta)=\partial_{\zeta_{1}} \tilde{G}_{k}(z, \zeta)=0 & \text { for } \zeta_{1}=0 .\end{cases}
$$

For $k \rightarrow \infty$, using [1], we find $\tilde{G}_{z}=\tilde{G}(z,.) \in C^{4}\left(\overline{\mathbb{R}_{-}^{n}} \backslash\{z\}\right)$ such that

$$
\tilde{G}_{k}(z, .) \rightarrow \tilde{G}_{z} \text { in } C_{l o c}^{4}\left(\overline{\mathbb{R}_{-}^{n}} \backslash\{z\}\right), \quad \Delta_{g_{\infty}, \zeta}^{2} \tilde{G}(z, \zeta)=0(z \neq \zeta)
$$

$$
|\tilde{G}(z, \zeta)| \leq C \cdot \begin{cases}|z-\zeta|^{4-n}, & \text { if } n>4, \\ (1+|\log | z-\zeta||+\log (1+|z|)+\log (1+|\zeta|)), & \text { if } n=4, \\ (1+|z|+|\zeta|), & \text { if } n=3\end{cases}
$$

$$
|\nabla \tilde{G}(z, \zeta)| \leq C \cdot \begin{cases}|z-\zeta|^{-1}, & \text { if } n=4 \\ 1, & \text { if } n=3\end{cases}
$$

In order to calculate the differential equation satisfied by $\tilde{G}$ near $\zeta=z$, we introduce

$$
\varphi \in C_{c}^{\infty}\left(\overline{\mathbb{R}_{-}^{n}}\right), \quad \varphi=\partial_{1} \varphi=0 \text { on } \partial \mathbb{R}_{-}^{n}
$$

and let $\varphi_{k} \in C^{4, \alpha}\left(\overline{\Omega_{k}}\right)$ such that

$$
\begin{gathered}
\varphi(z)=\varphi_{k} \circ \Phi_{k, i}\left(x_{k}^{\prime}+\left|x_{k}^{\prime}-y_{k}^{\prime}\right|\left(z-\rho_{k} \vec{e}_{1}\right)\right) \text { for } z \in \tilde{\Omega}_{k} \\
\varphi_{k}=\partial_{\nu} \varphi_{k}=0 \text { on } \partial \Omega_{k} ;
\end{gathered}
$$

where we denote

$$
\tilde{\Omega}_{k}:=\rho_{k} \vec{e}_{1}-\frac{x_{k}^{\prime}}{\left|x_{k}^{\prime}-y_{k}^{\prime}\right|}+\frac{1}{\left|x_{k}^{\prime}-y_{k}^{\prime}\right|}\left(U_{i} \cap \mathbb{R}_{-}^{n}\right) .
$$


By means of the representation formula and the corresponding Green's function we see that for $z \in \mathbb{R}_{-}^{n}$ and $k$ large enough

$$
\begin{aligned}
\varphi(z)= & \varphi_{k}\left(\Phi_{k, i}\left(x_{k}^{\prime}+\left|x_{k}^{\prime}-y_{k}^{\prime}\right|\left(z-\rho_{k} \vec{e}_{1}\right)\right)\right) \\
= & \int_{\Omega_{k}} G_{k}\left(\Phi_{k, i}\left(x_{k}^{\prime}+\left|x_{k}^{\prime}-y_{k}^{\prime}\right|\left(z-\rho_{k} \vec{e}_{1}\right)\right), y\right)\left(\Delta^{2} \varphi_{k}+a_{k} \varphi_{k}\right) d y \\
= & \int_{\Phi_{k, i}\left(U_{i} \cap\left\{\eta_{1}<0\right\}\right)} G_{k}\left(\Phi_{k, i}\left(x_{k}^{\prime}+\left|x_{k}^{\prime}-y_{k}^{\prime}\right|\left(z-\rho_{k} \vec{e}_{1}\right)\right), y\right)\left(\Delta^{2} \varphi_{k}+a_{k} \varphi_{k}\right) d y \\
= & \int_{U_{i} \cap\left\{\eta_{1}<0\right\}} G_{k}\left(\Phi_{k, i}\left(x_{k}^{\prime}+\left|x_{k}^{\prime}-y_{k}^{\prime}\right|\left(z-\rho_{k} \vec{e}_{1}\right)\right), \Phi_{k, i}(\eta)\right) \\
& \cdot\left(\Delta_{\Phi_{k, i}^{*}(\mathcal{E})}^{2}\left(\varphi_{k} \circ \Phi_{k, i}\right)+\left(a_{k} \circ \Phi_{k, i}\right)\left(\varphi_{k} \circ \Phi_{k, i}\right)\right)(\eta)\left|\mathrm{Jac} \Phi_{k, i}(\eta)\right| d \eta \\
= & \int_{\tilde{\Omega}_{k}}\left|x_{k}^{\prime}-y_{k}^{\prime}\right|^{4-n} \tilde{G}_{k}(z, \zeta)\left|x_{k}^{\prime}-y_{k}^{\prime}\right|^{-4} \\
& \cdot\left(\Delta_{g_{k, i}}^{2}+\left|x_{k}^{\prime}-y_{k}^{\prime}\right|^{4} a_{k}\left(\Phi_{k, i}\left(x_{k}^{\prime}+\left|x_{k}^{\prime}-y_{k}^{\prime}\right|\left(\zeta-\rho_{k} \vec{e}_{1}\right)\right)\right)\right) \varphi(\zeta) \\
= & \quad \cdot\left|x_{k}^{\prime}-y_{k}^{\prime}\right|^{n}\left|\mathrm{Jac}_{k, i}\left(x_{k}^{\prime}+\left|x_{k}^{\prime}-y_{k}^{\prime}\right|\left(\zeta-\rho_{k} \vec{e}_{1}\right)\right)\right| d \zeta \\
\tilde{\Omega}_{k}(z, \zeta) & \cdot\left(\Delta_{g_{k, i}}^{2}+\left|x_{k}^{\prime}-y_{k}^{\prime}\right|^{4} a_{k}\left(\Phi_{k, i}\left(x_{k}^{\prime}+\left|x_{k}^{\prime}-y_{k}^{\prime}\right|\left(\zeta-\rho_{k} \vec{e}_{1}\right)\right)\right)\right) \varphi(\zeta) \\
& \cdot\left|\mathrm{Jac}_{k, i}\left(x_{k}^{\prime}+\left|x_{k}^{\prime}-y_{k}^{\prime}\right|\left(\zeta-\rho_{k} \vec{e}_{1}\right)\right)\right| d \zeta .
\end{aligned}
$$

Observing (57), (58) and passing to the limit we obtain for $z \in \mathbb{R}_{-}^{n}$ :

$$
\varphi(z)=\int_{\mathbb{R}_{-}^{n}} \tilde{G}(z, \zeta) \Delta_{g_{\infty, i}}^{2} \varphi(\zeta)\left|\operatorname{Jac} \Phi_{i}\left(x_{\infty}^{\prime}\right)\right| d \zeta .
$$

We introduce the linear bijection $L=d \Phi_{i}\left(x_{\infty}^{\prime}\right)$, the half space $P:=L\left(\mathbb{R}_{-}^{n}\right)$ and obtain for $z \in \mathbb{R}_{-}^{n}$ :

$$
\varphi(z)=\int_{\mathbb{R}_{-}^{n}} \tilde{G}(z, \zeta) \Delta_{L^{*} \mathcal{E}}^{2} \varphi(\zeta)|\operatorname{det}(L)| d \zeta=\int_{P} \tilde{G}\left(z, L^{-1}(\eta)\right) \Delta^{2}\left(\varphi \circ L^{-1}\right) d \eta
$$

Finally we consider a rotation $\sigma \in O(n)$ such that $\sigma(P)=\mathbb{R}_{-}^{n}$ so that $\sigma \circ L\left(\mathbb{R}_{-}^{n}\right)=$ $\mathbb{R}_{-}^{n}$. For arbitrary

$$
\psi \in C_{c}^{\infty}\left(\overline{\mathbb{R}_{-}^{n}}\right), \quad \text { with } \psi=\partial_{1} \psi=0 \text { on } \partial \mathbb{R}_{-}^{n}
$$

and $\tilde{x} \in \mathbb{R}_{-}^{n}$ we may take $\varphi=\psi \circ \sigma \circ L$ and $z=(\sigma \circ L)^{-1}(\tilde{x})$. We obtain from (64) since the Laplacian is invariant under orthogonal transformations that for $\tilde{x} \in \mathbb{R}_{-}^{n}$ :

$$
\begin{aligned}
\psi(\tilde{x}) & =(\psi \circ \sigma \circ L)\left((\sigma \circ L)^{-1}(\tilde{x})\right) \\
& =\int_{P=\sigma^{-1}\left(\mathbb{R}_{-}^{n}\right)} \tilde{G}\left((\sigma \circ L)^{-1}(\tilde{x}), L^{-1}(\eta)\right) \Delta^{2}(\psi \circ \sigma)(\eta) d \eta \\
& =\int_{\mathbb{R}_{-}^{n}} \tilde{G}\left((\sigma \circ L)^{-1}(\tilde{x}),(\sigma \circ L)^{-1}(\eta)\right) \Delta^{2} \psi(\eta) d \eta .
\end{aligned}
$$

This shows that in the sense of distributions

$$
\Delta_{\tilde{y}}^{2} \bar{G}(\tilde{x}, .)=\delta_{\tilde{x}},
$$


where we have defined

$$
\bar{G}(\tilde{x}, \tilde{y}):=\tilde{G}\left((\sigma \circ L)^{-1}(\tilde{x}),(\sigma \circ L)^{-1}(\tilde{y})\right) .
$$

Moreover, for fixed $\tilde{x} \in \mathbb{R}_{-}^{n}$ one concludes with the help of (62) and (63) that

$$
|\bar{G}(\tilde{x}, \tilde{y})| \leq C \cdot \begin{cases}|\tilde{x}-\tilde{y}|^{4-n}, & \text { if } n>4 \\ (1+|\log | \tilde{x}-\tilde{y}||+\log (1+|\tilde{x}|)+\log (1+|\tilde{y}|)), & \text { if } n=4 \\ (1+|\tilde{x}|+|\tilde{y}|), & \text { if } n=3\end{cases}
$$

$$
|\nabla \bar{G}(\tilde{x}, \tilde{y})| \leq C \cdot \begin{cases}|\tilde{x}-\tilde{y}|^{-1}, & \text { if } n=4 \\ 1, & \text { if } n=3 .\end{cases}
$$

We denote by $H$ the biharmonic Green's function in $\mathbb{R}_{-}^{n}$, which thanks to Boggio 4 is known explicitly and known to be positive - see Lemma 6.6 below. We prove:

Lemma 6.5. $\forall x, y \in \mathbb{R}_{-}^{n}, x \neq y: \quad \bar{G}(x, y)=H(x, y)$.

Proof. In what follows we keep $x \in \mathbb{R}_{-}^{n}$ fixed. Both $\bar{G}(x,$.$) and H(x,$.$) satisfy the$ biharmonic equation with the $\delta$-distribution $\delta_{x}$ as right hand side and zero Dirichlet boundary conditions on $\left\{y_{1}=0\right\}$. We let $\psi_{x}:=\bar{G}(x,)-.H(x,$.$) . Hence,$

$$
\psi=\psi_{x} \in C^{\infty}\left(\overline{\mathbb{R}_{-}^{n}}\right)
$$

solves

$$
\begin{cases}\Delta^{2} \psi=0 & \text { in } \mathbb{R}_{-}^{n} \\ \psi=\partial_{1} \psi=0 & \text { on }\left\{y_{1}=0\right\}\end{cases}
$$

Moreover, according to (67,68) and (77) below we have that

$$
\begin{array}{r}
\forall y \in \mathbb{R}_{-}^{n}: \quad|\psi(y)| \leq C \cdot \begin{cases}|y|^{4-n}, & \text { if } n>4, \\
(1+|\log | y||), & \text { if } n=4, \\
(1+|y|), & \text { if } n=3 ;\end{cases} \\
|\nabla \psi(y)| \leq C \cdot \begin{cases}|y|^{-1}, & \text { if } n=4, \\
1, & \text { if } n=3 ;\end{cases}
\end{array}
$$

where $C=C(x)$. According to [10, 20]

$$
\psi^{*}(y):= \begin{cases}\psi(y) & \text { if } y_{1} \leq 0 \\ -\psi\left(-y_{1}, \bar{y}\right)-2 y_{1} \frac{\partial}{\partial y_{1}} \psi\left(-y_{1}, \bar{y}\right)-y_{1}^{2} \Delta \psi\left(-y_{1}, \bar{y}\right), & \text { if } y_{1}>0\end{cases}
$$

$\psi^{*} \in C^{4}\left(\mathbb{R}^{n}\right)$ is an entire biharmonic function. We consider now first the case $n>4$. Below we will prove that (69) and (70) imply that also

$$
\forall j=1,2: \forall y \in \mathbb{R}_{-}^{n}: \quad\left|\nabla^{j} \psi(y)\right| \leq C|y|^{4-n-j} \text {, where } C=C(x) .
$$

This immediately gives that $\left|\psi^{*}(y)\right| \leq C|y|^{4-n}$ and in particular that $\psi^{*}$ is a bounded entire biharmonic function. Again, Liouville's theorem for biharmonic functions [25, p. 19] yields that $\psi^{*}(y) \equiv 0$ so that the claim of the lemma follows, provided $n>4$.

If $n=3,4$ we shall prove below that for $j=0,1,2$

$$
\forall y \in \mathbb{R}_{-}^{n}: \quad\left|D^{2+j} \psi(y)\right| \leq C|y|^{2-n-j}, \quad \text { where } C=C(x) .
$$

As above $\psi^{*}$ is an entire biharmonic function and so are $D \psi^{*}$ and $D^{2} \psi^{*}$. Since $\left|D^{2} \psi^{*}(y)\right| \leq C(1+|y|)^{2-n}$, it follows that $D^{2} \psi^{*}(x) \equiv 0$. In view of the boundary conditions in (69) we come up with $\psi^{*}(y) \equiv 0$ also in the case $n=3,4$. 
It remains to prove (72) and (73). We consider first $n>4$. Assume by contradiction that there exists a sequence $\left(y_{\ell}\right) \subset \mathbb{R}_{-}^{n}$ such that $\left|\nabla^{j} \psi\left(y_{\ell}\right)\right| \cdot\left|y_{\ell}\right|^{n+j-4} \rightarrow \infty$ for $\ell \rightarrow \infty$. Then

$$
\tilde{\psi}_{\ell}(y):=\left|y_{\ell}\right|^{n-4} \psi\left(y_{\ell}-y_{\ell, 1} \vec{e}_{1}+\left|y_{\ell}\right| y\right)
$$

would solve

$$
\begin{cases}\Delta^{2} \tilde{\psi}_{\ell}=0 & \text { in } \mathbb{R}_{-}^{n} \\ \tilde{\psi}_{\ell}=\partial_{1} \tilde{\psi}_{\ell}=0 & \text { on }\left\{y_{1}=0\right\}\end{cases}
$$

From the assumption we conclude that

$$
\left|\nabla^{j} \tilde{\psi}_{\ell}\left(\frac{y_{\ell, 1}}{\left|y_{\ell}\right|} \vec{e}_{1}\right)\right|=\left|y_{\ell}\right|^{n+j-4}\left|\nabla^{j} \psi\left(y_{\ell}\right)\right| \rightarrow \infty .
$$

On the other hand,

$$
\left|\tilde{\psi}_{\ell}(y)\right| \leq C\left|y_{\ell}\right|^{n-4}\left|y_{\ell}-y_{\ell, 1} \vec{e}_{1}+\right| y_{\ell}|y|^{4-n} \leq C\left|\frac{y_{\ell}}{\left|y_{\ell}\right|}+y-\frac{y_{\ell, 1}}{\left|y_{\ell}\right|} \vec{e}_{1}\right|^{4-n}
$$

so that $\tilde{\psi}_{\ell}$ remains bounded in a neighbourhood of $\frac{y_{\ell, 1}}{\left|y_{\ell}\right|} \vec{e}_{1}$ in $\overline{\mathbb{R}_{-}^{n}}$. Local Schauder estimates [1, Theorem 7.3] yield

$$
\left|\nabla^{j} \tilde{\psi}_{\ell}\left(\frac{y_{\ell, 1}}{\left|y_{\ell}\right|} \vec{e}_{1}\right)\right| \leq C
$$

thereby contradicting (75). This proves (72).

As for (73), i.e. in particular $n=3,4$, the proof is quite similar since we can already make use of the gradient estimates (71). Instead of (76) one has to make use of

$$
\left|\nabla \tilde{\psi}_{\ell}(y)\right| \leq C\left|y_{\ell}\right|^{n-3}\left|y_{\ell}-y_{\ell, 1} \vec{e}_{1}+\right| y_{\ell}|y|^{3-n} \leq C\left|\frac{y_{\ell}}{\left|y_{\ell}\right|}+y-\frac{y_{\ell, 1}}{\left|y_{\ell}\right|} \vec{e}_{1}\right|^{3-n},
$$

so that $\nabla \tilde{\psi}_{\ell}$ remains bounded uniformly outside $\frac{y_{\ell}}{\left|y_{\ell}\right|}-\frac{y_{\ell, 1}}{\left|y_{\ell}\right|} \vec{e}_{1}$. Therefore, since $\tilde{\psi}_{\ell}$ vanishes on $\partial \mathbb{R}_{-}^{n}$, we get that $\tilde{\psi}_{\ell}$ is bounded in a neighbourhood of $\frac{y_{\ell, 1}}{\left|y_{\ell}\right|} \vec{e}_{1}$ in $\overline{\mathbb{R}_{-}^{n}}$. The proof of the present lemma is complete.

In order to show that the present case $x_{\infty}=y_{\infty} \in \partial \Omega$ cannot occur we collect some basic facts on the biharmonic Green's function in the half space; modulo a simple conformal transformation, cf. [4, p. 126]:

Lemma 6.6. The biharmonic Green's function in $\mathbb{R}_{-}^{n}$ is given by

$$
\forall x, y \in \mathbb{R}_{-}^{n}: H(x, y)=\frac{1}{4 n e_{n}}|x-y|^{4-n} \int_{1}^{\left|x^{*}-y\right| /|x-y|}\left(v^{2}-1\right) v^{1-n} d v
$$

where $x^{*}=\left(-x_{1}, \bar{x}\right)$. From this it follows by direct calculation:

$$
\begin{aligned}
\forall x, y \in \mathbb{R}_{-}^{n}, x \neq y: & H(x, y)>0 ; \\
\forall x \in \mathbb{R}_{-}^{n}, y \in \partial \mathbb{R}_{-}^{n}: & \Delta_{y} H(x, y)>0 ; \\
\forall x, y \in \partial \mathbb{R}_{-}^{n}, x \neq y: & \Delta_{x} \Delta_{y} H(x, y)>0 .
\end{aligned}
$$


We proceed by showing that $x_{\infty}=y_{\infty} \in \partial \Omega$ is indeed impossible and recall that by assumption we chose $x_{k}, y_{k}$ such that $G_{k}\left(x_{k}, y_{k}\right)=0$. In terms of the transformed Green's functions this reads

$$
\tilde{G}_{k}\left(\rho_{k} \vec{e}_{1}, \frac{y_{k}^{\prime}-x_{k}^{\prime}}{\left|x_{k}^{\prime}-y_{k}^{\prime}\right|}+\rho_{k} \vec{e}_{1}\right)=0
$$

cf. (59). After possibly extracting a further subsequence we find a point

$$
\theta=\lim _{k \rightarrow \infty} \frac{y_{k}^{\prime}-x_{k}^{\prime}}{\left|x_{k}^{\prime}-y_{k}^{\prime}\right|}
$$

and may conclude that

$$
\tilde{G}\left(\rho \vec{e}_{1}, \theta+\rho \vec{e}_{1}\right)=0 .
$$

According to the possible location of the limit points we have to distinguish four cases:

Case (a): $\rho<0$ and $\left(\theta+\rho \vec{e}_{1}\right)_{1}<0$. We put $\tilde{x}=(\sigma \circ L)\left(\rho \vec{e}_{1}\right) \in \mathbb{R}_{-}^{n}, \tilde{y}=$ $(\sigma \circ L)\left(\theta+\rho \vec{e}_{1}\right) \in \mathbb{R}_{-}^{n}$. According to [66) and Lemma 6.5 we could conclude that

$$
H(\tilde{x}, \tilde{y})=\bar{G}(\tilde{x}, \tilde{y})=0,
$$

which is impossible in view of (78).

Case (b): $\rho=0$ and $\left(\theta+\rho \vec{e}_{1}\right)_{1}<0$. As in the proof of Lemma 6.2 we conclude from (81) that $\partial_{x_{1}}^{2} \tilde{G}(0, \theta)=0$. Together with the Dirichlet boundary conditions satisfied by $\tilde{G}$ this yields $\tilde{G}(0, \theta)=0, D_{x} \tilde{G}(0, \theta)=0, D_{x}^{2} \tilde{G}(0, \theta)=0$. If we put $\tilde{y}=(\sigma \circ L)(\theta) \in \mathbb{R}_{-}^{n}$ this implies due to (66) that also $D_{x}^{2} \bar{G}(0, \tilde{y})=0$. In particular, we have that $\Delta_{x} H(0, \tilde{y})=\Delta_{x} \bar{G}(0, \tilde{y})=0$, which is impossible in view of (79).

Case $(c): \rho<0$ and $\left(\theta+\rho \vec{e}_{1}\right)_{1}=0$. Due to symmetry of the Green's function, this case is completely analogous to the previous one and hence impossible in view of (79).

Case $(d): \rho=0$ and $\left(\theta+\rho \vec{e}_{1}\right)_{1}=0$. As in the proof of Lemma 6.4 we conclude from (81) that $\partial_{x_{1}}^{2} \partial_{y_{1}}^{2} \tilde{G}(0, \theta)=0$. Here $\theta_{1}=0,|\theta|=1$. Thanks to the boundary conditions satisfied by $\tilde{G}$ this gives $\forall|\alpha| \leq 2,|\beta| \leq 2: \quad D_{x}^{\alpha} D_{y}^{\beta} \tilde{G}(0, \theta)=0$. Using again (66), we see that also $\forall|\alpha| \leq 2,|\beta| \leq 2$ : $\quad D_{x}^{\alpha} D_{y}^{\beta} \bar{G}(0, \tilde{y})=0$, where $\tilde{y}=$ $(\sigma \circ L)(\theta) \neq 0$. In particular, we come up with $\Delta_{x} \Delta_{y} H(0, \tilde{y})=\Delta_{x} \Delta_{y} \bar{G}(0, \tilde{y})=0$. This is impossible in view of (80).

Conclusion. In each case we finally deduced a contradiction so that $x_{\infty}=y_{\infty} \in$ $\partial \Omega$ is indeed impossible. The proof of Lemma 6.3 is complete.

6.5. Proof of Theorems 1, 2 and 3. Theorem 3 follows from the conclusions made in Subsections 6.1, 6.2 and 6.3.

In order to prove Theorem 1 we assume for contradiction that there exist a bounded $C^{4, \alpha}$-smooth domain $\Omega \subset \mathbb{R}^{n}$ and sequences $\left(x_{k}\right),\left(y_{k}\right) \subset \Omega, x_{k} \neq y_{k}$ with $H_{\Omega}\left(x_{k}, y_{k}\right) \leq 0$ and $\lim _{k \rightarrow \infty}\left|x_{k}-y_{k}\right|=0$. In view of the smoothness assumption, we see by working in local coordinate charts that after possibly passing to a subsequence and relabelling we find $\tilde{y}_{k} \in \Omega, x_{k} \neq \tilde{y}_{k}$ with $H_{\Omega}\left(x_{k}, \tilde{y}_{k}\right)=0$ and $\left|x_{k}-\tilde{y}_{k}\right| \rightarrow 0$ for $k \rightarrow \infty$. Application of Theorem 3 in the special case $\Omega_{k}=\Omega$, $a_{k}=0$ shows that this is impossible. This contradiction proves that there exists a $\delta=\delta(\Omega)>0$ such that $x, y \in \Omega, x \neq y, H_{\Omega}(x, y) \leq 0 \Rightarrow|x-y| \geq \delta$. Estimate (3) 
now follows directly from (36) while (4) is a consequence of (2), i.e. of DallAcqua and Sweers 8 .

In order to prove Theorem 2, we assume that no such $\varepsilon_{0}>0$ exists. In view of the remark after Theorem 2, we would have a neighbourhood $U$ of $\bar{B}, C^{4, \alpha_{-}}$ smooth diffeomorphisms $\psi_{k}: U \rightarrow \psi_{k}(U)$ and smooth domains $\Omega_{k}=\psi_{k}(B)$ with sign changing biharmonic Green's functions $H_{k}$. Hence, one of the alternatives described in Theorem 3 would occur for the biharmonic Green's function $H$ in the ball $B$. Since $H$ enjoys precisely the analogous properties of Lemma 6.6 (cf. [4, p. 126]), this is false; Theorem 2 follows.

\section{REFERENCES}

[1] Agmon, S., Douglis, A., Nirenberg, L: Estimates near the boundary for solutions of elliptic partial differential equations satisfying general boundary conditions. I. Commun. Pure Appl. Math. 12, 623-727 (1959).

[2] Bernis, F.: Change of sign of the solutions to some parabolic problems. In: V. Lakshmikantham (ed.), Nonlinear Analysis and Applications, 75-82. New York, Marcel Dekker (1987).

[3] Boggio, T.: Sull'equilibrio delle piastre elastiche incastrate. Rend. Acc. Lincei 10, 197-205 (1901).

[4] Boggio, T.: Sulle funzioni di Green d'ordine $m$. Rend. Circ. Mat. Palermo 20, 97-135 (1905).

[5] Coffman, C.V., Duffin, R.J.: On the structure of biharmonic functions satisfying the clamped condition on a right angle. Adv. Appl. Math. 1, (1950), 373-389.

[6] Coffman, C.V., Grover, C.L.: Obtuse cones in Hilbert spaces and applications to partial differential equations. J. Funct. Anal. 35, 369-396 (1980).

[7] Dall'Acqua, A., Meister, Ch., Sweers, G.: Separating positivity and regularity for fourth order Dirichlet problems in 2d-domains. Analysis 25, 205-261 (2005).

[8] Dall'Acqua, A. Sweers, G.: Estimates for Green function and Poisson kernels of higher order Dirichlet boundary value problems. J. Differential Equations 205, 466-487 (2004).

[9] Duffin, R.J.: On a question of Hadamard concerning super-biharmonic functions. J. Math. Phys. 27, 253-258 (1949).

[10] Duffin, R.J.: Continuation of biharmonic functions by reflection. Duke Math. J. 22, 313-324 (1955).

[11] Garabedian, P.R.: A partial differential equation arising in conformal mapping. Pacific J. Math. 1, 485-524 (1951).

[12] Giraud, G.: Sur le problème de Dirichlet généralisé. Ann. Sc. Ecole Norm. Sup. 46, 131-245 (1929).

[13] Grunau, H.-Ch., Robert, F.: Boundedness of the negative part of biharmonic Green's functions under Dirichlet boundary conditions in general domains. C. R. Acad. Sci. Paris, Ser. I, 347 (2009).

[14] Grunau, H.-Ch., Sweers, G.: Positivity for perturbations of polyharmonic operators with Dirichlet boundary conditions in two dimensions. Math. Nachr. 179, 89-102 (1996).

[15] Grunau, H.-Ch., Sweers, G.: Positivity for equations involving polyharmonic operators with Dirichlet boundary conditions. Math. Ann. 307, 589-626 (1997).

[16] Grunau, H.-Ch., Sweers, G.: Regions of positivity for polyharmonic Green functions in arbitrary domains. Proc. Amer. Math. Society 135, 3537-3546 (2007).

[17] Hadamard, J.: Mémoire sur le problème d'analyse relatif à l'équilibre des plaques élastiques encastrées. In: Euvres de Jacques Hadamard, Tome II, 515-641. CNRS, Paris (1968).

[18] Hadamard, J.: Sur certains cas intéressants du problème biharmonique. In: Euvres de Jacques Hadamard, Tome III, 1297-1299. CNRS, Paris (1968).

[19] Hedenmalm, H., Jakobsson, S., Shimorin, S.: A biharmonic maximum principle for hyperbolic surfaces. J. Reine Angew. Math. 550, 25-75 (2002).

[20] Huber, A.: On the reflection principle for polyharmonic functions. Commun. Pure Appl. Math. 9, 471-478 (1956).

[21] Kawohl, B., Sweers, G.: On 'anti'-eigenvalues for elliptic systems and a question of McKenna and Walter. Indiana Univ. Math. J. 51, 1023-1040 (2002). 
[22] KrasovskiĬ, Ju.P.: Investigation of potentials connected with boundary value problems for elliptic equations (Russian). Izv. Akad. Nauk SSSR Ser. Mat. 31, 587-640 (1967). English translation in: Math. USSR, Izv. 1, 569-622 (1967).

[23] KrasovskiI, Ju.P.: Isolation of singularities of the Green's function (Russian). Izv. Akad. Nauk SSSR Ser. Mat. 31, 977-1010 (1967). English translation in: Math. USSR, Izv. 1, 935-966 (1967).

[24] Nehari, Z.: On the biharmonic Green's function. In: Studies Math. Mech., presented to Richard von Mises, 111-117. Academic Press, New York etc. (1954).

[25] Nicolesco, M.: Les fonctions polyharmoniques. Hermann, Paris (1936).

[26] Sassone, E.: Positivity for polyharmonic problems on domains close to a disk. Ann. Mat. Pura Appl. 186, 419-432 (2007).

[27] SCHRÖDER, J.: Randwertaufgaben vierter Ordnung mit positiver Greenscher Funktion. Math. Z. 90, 429-440 (1965).

[28] Schröder, J.: On linear differential inequalities. J. Math. Anal. Appl. 22, 188-216 (1968).

[29] SchröDER, J.: Operator inequalities. Math. Science Engineering 147. Academic Press, New York etc. (1980).

[30] Shapiro, H.S., Tegmark, M.: An elementary proof that the biharmonic Green function of an eccentric ellipse changes sign. SIAM Rev. 36, 99-101 (1994).

Fakultät für Mathematik, Otto-von-Guericke-Universität, Postfach 4120, 39016 MagdeBURG, GERMANY

E-mail address: hans-christoph.grunau@ovgu.de

Université de Nice-Sophia Antipolis, Laboratoire J.-A.Dieudonné, Parc Valrose, 06108 Nice Cedex 2, France

E-mail address: frobert@math.unice.fr 